\title{
Vacuum induced CP violation generating a complex CKM matrix with controlled scalar FCNC
}

\author{
Miguel Nebot ${ }^{1, \mathrm{a}}$, Francisco J. Botella ${ }^{2, \mathrm{~b}}$, Gustavo C. Branco ${ }^{1, \mathrm{c}}$ \\ ${ }^{1}$ Departamento de Física and Centro de Física Teórica de Partículas (CFTP), Instituto Superior Técnico (IST), U. de Lisboa (UL), \\ Av. Rovisco Pais 1, 1049-001 Lisbon, Portugal \\ ${ }^{2}$ Departament de Fìsica Teòrica and Instituto de Física Corpuscular (IFIC), Universitat de València-CSIC, 46100 Valencia, Spain
}

Received: 27 June 2019 / Accepted: 11 August 2019 / Published online: 23 August 2019

(C) The Author(s) 2019

\begin{abstract}
We propose a viable minimal model with spontaneous $\mathrm{CP}$ violation in the framework of a two Higgs doublet model. The model is based on a generalised Branco-GrimusLavoura model with a flavoured $\mathbb{Z}_{2}$ symmetry, under which two of the quark families are even and the third one is odd. The lagrangian respects $\mathrm{CP}$ invariance, but the vacuum has a $\mathrm{CP}$ violating phase, which is able to generate a complex CKM matrix, with the rephasing invariant strength of $\mathrm{CP}$ violation compatible with experiment. The question of scalar mediated flavour changing neutral couplings is carefully studied. In particular we point out a deep connection between the generation of a complex CKM matrix from a vacuum phase and the appearance of scalar FCNC. The scalar sector is presented in detail, showing that the new scalars are necessarily lighter than $1 \mathrm{TeV}$. A complete analysis of the model including the most relevant constraints is performed, showing that it is viable and that it has definite implications for the observation of New Physics signals in, for example, flavour changing Higgs decays or the discovery of the new scalars at the LHC. We give special emphasis to processes like $t \rightarrow \mathrm{h} c, \mathrm{~h} u$, as well as $\mathrm{h} \rightarrow b s, b d$, which are relevant for the LHC and the ILC.
\end{abstract}

\section{Introduction}

The first model of spontaneous $\mathrm{T}$ and $\mathrm{CP}$ violation was proposed [1] by Lee in 1973 at a time when only two incomplete quark generations were known. The main motivation for Lee's seminal work was to put the breaking of $\mathrm{CP}$ and $\mathrm{T}$ on the same footing as the breaking of gauge symmetry. In Lee's model, the Lagrangian is $\mathrm{CP}$ and $\mathrm{T}$ invariant, but the vac-

\footnotetext{
a e-mail: miguel.r.nebot.gomez@tecnico.ulisboa.pt

be-mail: Francisco.J.Botella@uv.es

ce-mail: gbranco@tecnico.ulisboa.pt
}

uum violates these discrete symmetries. This was achieved through the introduction of two Higgs doublets, with vacuum expectation values with a relative phase which violates $\mathrm{T}$ and $\mathrm{CP}$ invariance. In Lee's model, $\mathrm{CP}$ violation would arise solely from Higgs exchange, since at the time only two generations were known and therefore the CKM matrix was real. The general two Higgs Doublet Model (2HDM) [2,3] has Scalar Flavour Changing Neutral Couplings (SFCNC) at tree level which need to be controlled in order to conform to the stringent experimental constraints. This can be achieved by imposing Natural Flavour Conservation (NFC) in the scalar sector, as suggested by Glashow and Weinberg (GW) [4]. Alternatively, it was suggested by Branco, Grimus and Lavoura (BGL) [5] that one may have 2HDM with tree level SFCNC but with their flavour structure only dependent on the CKM matrix $V$.

BGL models have been extensively analysed in the literature [6-11], and their phenomenological consequences have been studied, in particular in the context of LHC. Recently BGL models have been generalised [12] in the framework of 2HDM. Both the GW and the BGL schemes can be implemented through the introduction of extra symmetries in the 2HDM. On the other hand, it has been shown [13] that the introduction of these symmetries in the 2HDM prevents the generation of either spontaneous or explicit $\mathrm{CP}$ violation in the scalar sector, unless they are softly broken [14]. It was recently discussed [15] that for a scalar potential with an extra symmetry beyond gauge symmetry, there is an intriguing correlation between the capability of the potential to generate explicit and spontaneous $\mathrm{CP}$ violation.

In this paper we propose a realistic model of spontaneous $\mathrm{CP}$ violation in the framework of $2 \mathrm{HDM}$. At this stage it is worth recalling the obstacles which have to be surmounted 
by any model of spontaneous CP violation that we are mainly interested here ${ }^{1}$ :

(i) The scalar potential should be able to generate spontaneous CP breaking by a phase of the vacuum, denoted $\theta$.

(ii) The phase $\theta$ should be able to generate a complex CKM matrix, with the strength of $\mathrm{CP}$ violation compatible with experiment. Recall that the CKM matrix has to be complex even in the presence of New Physics [17].

(iii) SFCNC effects should be under control so that they do not violate experimental bounds.

The origin of $\mathrm{CP}$ violation is a fundamental open question in Particle Physics. In particular, one does not know whether CP is explicitly broken at the Lagrangian level, as in the Standard Model (SM) or it is a good symmetry of the Lagrangian, only violated by the vacuum. In order to address this question, one has to have a viable model of spontaneous $\mathrm{CP}$ violation, as the model which we propose in this paper. Another question which may also be addressed in the framework of spontaneous $\mathrm{CP}$ violation is the strong $\mathrm{CP}$ problem. The present paper has not the purpose of providing a solution to the strong $\mathrm{CP}$ problem. However, it is remarkable that $\theta_{\mathrm{QFD}}$ naturally vanishes at tree level in the model and it is a calculable quantity. At one loop one has to do some fine-tuning of parameters in order to have $\bar{\theta}$ sufficiently small. The level of fine-tuning is less severe than in the SM, where $\bar{\theta}$ is an arbitrary parameter of order one, which has be be fine-tuned to be less than $10^{-10}$.

The paper is organised as follows. In the next section we present the structure of the model and specify the flavoured symmetry introduced. In the third section we show how a complex CKM matrix is generated from the vacuum phase. Section 4 contains a detailed analysis of the scalar potential with real couplings. In Sect. 5 we derive the physical Yukawa couplings and the phenomenological analysis of the model is presented in Sect. 6. Finally we present our conclusions in the last section.

\section{The structure of the model and the flavoured symmetry}

The Yukawa couplings in the 2HDM read

$\mathscr{L}_{\mathrm{Y}}=-\bar{Q}_{L}^{0}\left(\Gamma_{1} \Phi_{1}+\Gamma_{2} \Phi_{2}\right) d_{R}^{0}-\bar{Q}_{L}^{0}\left(\Delta_{1} \tilde{\Phi}_{1}+\Delta_{2} \tilde{\Phi}_{2}\right) u_{R}^{0}+$ H.c.

\footnotetext{
1 A general and detailed enumeration of theoretical challenges can found in [16].
}

with summation over generation indices understood and $\tilde{\Phi}_{j}=i \sigma_{2} \Phi_{j}^{*}$. We consider the following $\mathbb{Z}_{2}$ transformations to define the model:

$\Phi_{1} \mapsto \Phi_{1}, \quad \Phi_{2} \mapsto-\Phi_{2}, \quad Q_{L 3}^{0} \mapsto-Q_{L 3}^{0}$,

$Q_{L j}^{0} \mapsto Q_{L j}^{0}, \quad j=1,2$,

$d_{R k}^{0} \mapsto d_{R k}^{0}, \quad u_{R k}^{0} \mapsto u_{R k}^{0}, \quad k=1,2,3$.

Invariance under Eq. (2) gives the following form of the Yukawa coupling matrices:

$$
\begin{aligned}
\Gamma_{1}=\left(\begin{array}{ccc}
\times & \times & \times \\
\times & \times & \times \\
0 & 0 & 0
\end{array}\right), & \Gamma_{2}=\left(\begin{array}{ccc}
0 & 0 & 0 \\
0 & 0 & 0 \\
\times & \times & \times
\end{array}\right), \\
\Delta_{1}=\left(\begin{array}{ccc}
\times & \times & \times \\
\times & \times & \times \\
0 & 0 & 0
\end{array}\right), & \Delta_{2}=\left(\begin{array}{ccc}
0 & 0 & 0 \\
0 & 0 & 0 \\
\times & \times & \times
\end{array}\right) .
\end{aligned}
$$

The symmetry assignment in Eq. (2) and the Yukawa matrices in Eq. (3) correspond to the generalised BGL models introduced in [12]. We impose CP invariance at the Lagrangian level, so we require the Yukawa couplings to be real:

$\Gamma_{j}^{*}=\Gamma_{j}, \quad \Delta_{j}^{*}=\Delta_{j}$

We write the scalar doublets $\Phi_{j}$ in the "Higgs basis" $\left\{H_{1}, H_{2}\right\}$ [18-20] (see Sect. 4 and Appendix B for further details on the scalar sector)

$$
\begin{aligned}
& \left(\begin{array}{l}
H_{1} \\
H_{2}
\end{array}\right)=\mathcal{R}_{\beta}\left(\begin{array}{l}
e^{-i \theta_{1}} \Phi_{1} \\
e^{-i \theta_{2}} \Phi_{2}
\end{array}\right), \text { with } \mathcal{R}_{\beta}=\left(\begin{array}{cc}
c_{\beta} & s_{\beta} \\
-s_{\beta} & c_{\beta}
\end{array}\right), \\
& \mathcal{R}_{\beta}^{T}=\mathcal{R}_{\beta}^{-1} .
\end{aligned}
$$

In this basis, only $H_{1}$ acquires a vacuum expectation value

$$
\left\langle H_{1}\right\rangle=\frac{v}{\sqrt{2}}\left(\begin{array}{l}
0 \\
1
\end{array}\right), \quad\left\langle H_{2}\right\rangle=\left(\begin{array}{l}
0 \\
0
\end{array}\right) \text {. }
$$

Equation (1) can then be rewritten as

$$
\begin{aligned}
\mathscr{L}_{\mathrm{Y}}= & -\frac{\sqrt{2}}{v} \bar{Q}_{L}^{0}\left(M_{d}^{0} H_{1}+N_{d}^{0} H_{2}\right) d_{R}^{0} \\
& -\frac{\sqrt{2}}{v} \bar{Q}_{L}^{0}\left(M_{u}^{0} \tilde{H}_{1}+N_{u}^{0} \tilde{H}_{2}\right) u_{R}^{0}+\text { H.c. },
\end{aligned}
$$

where the quark mass matrices $M_{d}^{0}, M_{u}^{0}$ and the $N_{d}^{0}, N_{u}^{0}$ matrices read

$M_{d}^{0}=\frac{v e^{i \theta_{1}}}{\sqrt{2}}\left(c_{\beta} \Gamma_{1}+e^{i \theta} s_{\beta} \Gamma_{2}\right)$,

$N_{d}^{0}=\frac{v e^{i \theta_{1}}}{\sqrt{2}}\left(-s_{\beta} \Gamma_{1}+e^{i \theta} c_{\beta} \Gamma_{2}\right)$,

$M_{u}^{0}=\frac{v e^{-i \theta_{1}}}{\sqrt{2}}\left(c_{\beta} \Delta_{1}+e^{-i \theta} s_{\beta} \Delta_{2}\right)$, 
$N_{u}^{0}=\frac{v e^{-i \theta_{1}}}{\sqrt{2}}\left(-s_{\beta} \Delta_{1}+e^{-i \theta} c_{\beta} \Delta_{2}\right)$,

where $\theta=\theta_{2}-\theta_{1}$ is the relative phase among $\left\langle\Phi_{2}\right\rangle$ and $\left\langle\Phi_{1}\right\rangle$. For simplicity, we remove the irrelevant global phases $e^{ \pm i \theta_{1}}$ setting $\theta_{1}=0$.

Notice that the matrices $N_{d}^{0}$, $N_{u}^{0}$ can be written:

$$
\begin{aligned}
N_{d}^{0} & =t_{\beta} M_{d}^{0}+e^{i \theta} \frac{v}{\sqrt{2}}\left(t_{\beta}+t_{\beta}^{-1}\right) s_{\beta} \Gamma_{2} \\
& =t_{\beta} M_{d}^{0}-\left(t_{\beta}+t_{\beta}^{-1}\right) \mathrm{P}_{3} M_{d}^{0}, \\
N_{u}^{0} & =t_{\beta} M_{u}^{0}+e^{-i \theta} \frac{v}{\sqrt{2}}\left(t_{\beta}+t_{\beta}^{-1}\right) s_{\beta} \Delta_{2} \\
& =t_{\beta} M_{u}^{0}-\left(t_{\beta}+t_{\beta}^{-1}\right) \mathrm{P}_{3} M_{u}^{0},
\end{aligned}
$$

where $\mathrm{P}_{3}$ is the projector

$$
P_{3}=\left(\begin{array}{lll}
0 & 0 & 0 \\
0 & 0 & 0 \\
0 & 0 & 1
\end{array}\right) \text {. }
$$

\section{Generation of a complex CKM matrix from the vacuum phase}

In this section, we show how the vacuum phase $\theta$ is capable of generating a complex CKM matrix. As previously emphasized, this is a necessary requirement for the model to be consistent with experiment. Following Eqs. (3), (4) and (8), (9), we write:

$M_{d}^{0}=\left(\begin{array}{ccc}1 & 0 & 0 \\ 0 & 1 & 0 \\ 0 & 0 & e^{i \theta}\end{array}\right) \hat{M}_{d}^{0}, \quad M_{u}^{0}=\left(\begin{array}{ccc}1 & 0 & 0 \\ 0 & 1 & 0 \\ 0 & 0 & e^{-i \theta}\end{array}\right) \hat{M}_{u}^{0}$,

with $\hat{M}_{d}^{0}$ and $\hat{M}_{u}^{0}$ real. Then,

$M_{d}^{0} M_{d}^{0 \dagger}=\left(\begin{array}{ccc}1 & 0 & 0 \\ 0 & 1 & 0 \\ 0 & 0 & e^{i \theta}\end{array}\right) \hat{M}_{d}^{0} \hat{M}_{d}^{0 T}\left(\begin{array}{ccc}1 & 0 & 0 \\ 0 & 1 & 0 \\ 0 & 0 & e^{-i \theta}\end{array}\right)$

with $\hat{M}_{d}^{0} \hat{M}_{d}^{0 T}$ real and symmetric, which is diagonalised with a real orthogonal transformation:

$\mathcal{O}_{L}^{d T} \hat{M}_{d}^{0} \hat{M}_{d}^{0 T} \mathcal{O}_{L}^{d}=\operatorname{diag}\left(m_{d_{i}}^{2}\right)$.

Consequently, Eq. (14) gives

$\mathcal{O}_{L}^{d T}\left(\begin{array}{ccc}1 & 0 & 0 \\ 0 & 1 & 0 \\ 0 & 0 & e^{-i \theta}\end{array}\right) M_{d}^{0} M_{d}^{0 \dagger}\left(\begin{array}{lll}1 & 0 & 0 \\ 0 & 1 & 0 \\ 0 & 0 & e^{i \theta}\end{array}\right) \mathcal{O}_{L}^{d}=\operatorname{diag}\left(m_{d_{i}}^{2}\right)$.

That is, the diagonalisation of $M_{d}^{0} M_{d}^{0 \dagger}$ is accomplished with

$\mathcal{U}_{L}^{d \dagger} M_{d}^{0} M_{d}^{0 \dagger} \mathcal{U}_{L}^{d}=\operatorname{diag}\left(m_{d_{i}}^{2}\right), \quad$ where $\mathcal{U}_{L}^{d}=\left(\begin{array}{lll}1 & 0 & 0 \\ 0 & 1 & 0 \\ 0 & 0 & e^{i \theta}\end{array}\right) \mathcal{O}_{L}^{d}$.
Similarly,

$\mathcal{U}_{L}^{u \dagger} M_{u}^{0} M_{u}^{0 \dagger} \mathcal{U}_{L}^{u}=\operatorname{diag}\left(m_{u_{i}}^{2}\right), \quad$ with $\mathcal{U}_{L}^{u}=\left(\begin{array}{ccc}1 & 0 & 0 \\ 0 & 1 & 0 \\ 0 & 0 & e^{-i \theta}\end{array}\right) \mathcal{O}_{L}^{u}$

Notice the important sign difference in $\theta$ between Eqs. (17) and (18), which give the following CKM matrix $V \equiv \mathcal{U}_{L}^{u \dagger} \mathcal{U}_{L}^{d}$,

$V=\mathcal{O}_{L}^{u T}\left(\begin{array}{lll}1 & 0 & 0 \\ 0 & 1 & 0 \\ 0 & 0 & e^{i 2 \theta}\end{array}\right) \mathcal{O}_{L}^{d}$.

Notice also that, if $e^{i 2 \theta}= \pm 1, V$ is real, i.e. it does not generate $\mathrm{CP}$ violation. This can be understood through a careful analysis of the potential, which will be presented in Sect. 4 . The model we present here has spontaneous $\mathrm{CP}$ violation and thus a physical phase in the CKM matrix can only arise from $\theta$. In Sect. 4.1 we show that for $\theta=\pi / 2$ the vacuum is $\mathrm{CP}$ invariant and no $\mathrm{CP}$ violation can be generated in this model. In particular CKM is necessarily real for this value of $\theta$, as noticed in Eq. (19).

It is also straightforward to observe that $M_{d}^{0 \dagger} M_{d}^{0}$ and $M_{u}^{0 \dagger} M_{u}^{0}$ are real and symmetric, and are thus diagonalised with real orthogonal matrices $\mathcal{O}_{R}^{d}$ and $\mathcal{O}_{R}^{u}$,

$\mathcal{O}_{R}^{d T} M_{d}^{0 \dagger} M_{d}^{0} \mathcal{O}_{R}^{d}=\operatorname{diag}\left(m_{d_{i}}^{2}\right)$,

$\mathcal{O}_{R}^{u T} M_{u}^{0 \dagger} M_{u}^{0} \mathcal{O}_{R}^{u}=\operatorname{diag}\left(m_{u_{i}}^{2}\right)$,

such that the bi-diagonalisation of $M_{d}^{0}$ and $M_{u}^{0}$ reads

$M_{d}=\operatorname{diag}\left(m_{d_{i}}\right)=\mathcal{U}_{L}^{d \dagger} M_{d}^{0} \mathcal{O}_{R}^{d}$,

$M_{u}=\operatorname{diag}\left(m_{u_{i}}\right)=\mathcal{U}_{L}^{u \dagger} M_{u}^{0} \mathcal{O}_{R}^{u}$.

Following Eq. (10),

$$
\begin{aligned}
N_{d} \equiv \mathcal{U}_{L}^{d \dagger} N_{d}^{0} \mathcal{O}_{R}^{d} & =t_{\beta} \mathcal{U}_{L}^{d \dagger} M_{d}^{0} \mathcal{O}_{R}^{d}-\left(t_{\beta}+t_{\beta}^{-1}\right) \mathcal{U}_{L}^{d \dagger} \mathrm{P}_{3} M_{d}^{0} \mathcal{O}_{R}^{d} \\
& =t_{\beta} M_{d}-\left(t_{\beta}+t_{\beta}^{-1}\right) \mathcal{U}_{L}^{d \dagger} \mathrm{P}_{3} \mathcal{U}_{L}^{d} M_{d}
\end{aligned}
$$

with $\mathrm{P}_{3}$ the projector in Eq. (12) and $\mathcal{U}_{L}^{d}$ in Eq. (17). In the last term of Eq. (22),

$\mathcal{U}_{L}^{d \dagger} \mathrm{P}_{3} \mathcal{U}_{L}^{d}=\mathcal{O}_{L}^{d T} \mathrm{P}_{3} \mathcal{O}_{L}^{d}$,

that is, $N_{d}$ in Eq. (22) is real. Introducing a real unit vector $\hat{r}_{\text {[d] }}$ and a complex unit vector $\hat{n}_{\text {[d] }}$ with components

$\hat{r}_{[\mathrm{d}] j} \equiv\left[\mathcal{O}_{L}^{d}\right]_{3 j}, \quad \hat{n}_{[\mathrm{d}] j} \equiv\left[\mathcal{U}_{L}^{d}\right]_{3 j}=e^{i \theta} \hat{r}_{[\mathrm{d}] j}$,

one has, for $\mathcal{U}_{L}^{d \dagger} \mathrm{P}_{3} \mathcal{U}_{L}^{d}$ in Eq. (23),

$\left[\mathcal{U}_{L}^{d \dagger} \mathrm{P}_{3} \mathcal{U}_{L}^{d}\right]_{i j}=\hat{n}_{[\mathrm{d}] i}^{*} \hat{n}_{[\mathrm{d}] j}=\hat{r}_{[\mathrm{d}]} \hat{r}_{[\mathrm{d}] j}$

Similarly, for $N_{u}$ we have

$N_{u} \equiv \mathcal{U}_{L}^{u \dagger} N_{u}^{0} \mathcal{O}_{R}^{u}=t_{\beta} M_{u}-\left(t_{\beta}+t_{\beta}^{-1}\right) \mathcal{U}_{L}^{u \dagger} \mathrm{P}_{3} \mathcal{U}_{L}^{u} M_{u}$, 
with

$\mathcal{U}_{L}^{u \dagger} \mathrm{P}_{3} \mathcal{U}_{L}^{u}=\mathcal{O}_{L}^{u T} \mathrm{P}_{3} \mathcal{O}_{L}^{u}$,

and

$\hat{r}_{[\mathrm{u}] j} \equiv\left[\mathcal{O}_{L}^{u}\right]_{3 j} \quad \hat{n}_{[\mathrm{u}] j} \equiv\left[\mathcal{U}_{L}^{u}\right]_{3 j}=e^{-i \theta} \hat{r}_{[\mathrm{u}] j}$,

$\left[\mathcal{U}_{L}^{u \dagger} \mathrm{P}_{3} \mathcal{U}_{L}^{u}\right]_{i j}=\hat{n}_{[\mathrm{u}] i}^{*} \hat{n}_{[\mathrm{u}] j}=\hat{r}_{[\mathrm{u}] i} \hat{r}_{[\mathrm{u}] j}$.

Like $N_{d}, N_{u}$ is real; $N_{d}$ and $N_{u}$ have the form:

$$
\begin{aligned}
& {\left[N_{d}\right]_{i j}=t_{\beta} \delta_{i j} m_{d_{i}}-\left(t_{\beta}+t_{\beta}^{-1}\right) \hat{n}_{[\mathrm{d}] i}^{*} \hat{n}_{[\mathrm{d}] j} m_{d_{j}},} \\
& {\left[N_{u}\right]_{i j}=t_{\beta} \delta_{i j} m_{u_{i}}-\left(t_{\beta}+t_{\beta}^{-1}\right) \hat{n}_{[\mathrm{u}] i}^{*} \hat{n}_{[\mathrm{u}] j} m_{u_{j}} .}
\end{aligned}
$$

Since $V=\mathcal{U}_{L}^{u^{\dagger}} \mathcal{U}_{L}^{d}$, the complex unitary vectors $\hat{n}_{[\mathrm{d}]}$ and $\hat{n}_{[\mathrm{u}]}$ are not independent:

$\hat{n}_{[\mathrm{d}] i}=\hat{n}_{[\mathrm{u}] j} V_{j i}, \quad \hat{n}_{[\mathrm{u}] i}=V_{i j}^{*} \hat{n}_{[\mathrm{d}] j}$.

It is interesting to notice that the 2HDM scenario studied in [21], where the soft breaking of a $\mathbb{Z}_{3}$ symmetry is the source of $\mathrm{CP}$ violation, shares some interesting properties with the present one: there, the CKM matrix can also be factorised in terms of real orthogonal rotations and a diagonal matrix containing the $\mathrm{CP}$ violating depence; the tree level SFCNC are also real in that phase convention. Other aspects of the model like the structure of the Yukawa couplings as well as the scalar sector to be discussed in Sect. 4 are, however, completely different.

In the rest of this section, we analyse in detail the generation of a complex CKM matrix from the vacuum phase $\theta$. The couplings of the physical scalars to the fermions are discussed in Sect. 5, after the discussion of the scalar sector in Sect. 4.

It is clear that $e^{i 2 \theta} \neq \pm 1$ is necessary in order to have an irreducibly complex CKM matrix. However, one has to verify that one can indeed obtain a realistic CKM matrix, one that it is in agreement with the experimental constraints on the moduli $\left|V_{i j}\right|$ (in particular of the moduli of the first and second rows), and on the CP violating phase $\gamma \equiv \arg \left(-V_{u d} V_{u b}^{*} V_{c b} V_{c d}^{*}\right)$ (the only one accessible through tree level processes alone). Concerning CP violation, one can alternatively analyse that the unique (up to a sign) imaginary part of a rephasing invariant quartet $\operatorname{Im}\left(V_{i_{1} j_{1}} V_{i_{1} j_{2}}^{*} V_{i_{2} j_{2}} V_{i_{2} j_{1}}^{*}\right)$ $\left(i_{1} \neq i_{2}, j_{1} \neq j_{2}\right)$ has the correct size $\sim 3 \times 10^{-5}$. Starting with Eq. (19), one can compute that imaginary part. For the task, it is convenient to trade $\mathcal{O}_{L}^{d}$ and $\mathcal{O}_{L}^{u}$ for the real unit vector $\hat{r}_{[\mathrm{d}]}$ in Eq. (24) and the real orthogonal matrix $R$ :

$\hat{r}_{[\mathrm{d}] j}=\left[\mathcal{O}_{L}^{d}\right]_{3 j}, \quad R \equiv \mathcal{O}_{L}^{u T} \mathcal{O}_{L}^{d}$.

Then, one can rewrite

$V=\mathcal{O}_{L}^{u T}\left[\mathbf{1}+\left(e^{i 2 \theta}-1\right) \mathrm{P}_{3}\right] \mathcal{O}_{L}^{d} \Rightarrow V_{i j}=R_{i j}+\left(e^{i 2 \theta}-1\right) S_{i j}$, and we introduce $S_{i j}$ to allow for compact expressions:

$$
\begin{aligned}
S_{i j} & \equiv\left[\mathcal{O}_{L}^{u T} \mathrm{P}_{3} \mathcal{O}_{L}^{d}\right]_{i j}=\hat{r}_{[\mathrm{u}] i} \hat{r}_{[\mathrm{d}] j}=\sum_{k=1}^{3} R_{i k} \hat{r}_{[\mathrm{d}] k} \hat{r}_{[\mathrm{d}] j} \\
& =\sum_{k=1}^{3} \hat{r}_{[\mathrm{u}] i} \hat{r}_{[\mathrm{u}] k} R_{k j} .
\end{aligned}
$$

The real and imaginary parts of $V_{i j}$ are ${ }^{2}$

$\operatorname{Re}\left(V_{i j}\right)=R_{i j}-2 s_{\theta}^{2} S_{i j}, \quad \operatorname{Im}\left(V_{i j}\right)=s_{2 \theta} S_{i j}$.

Notice that, although Eq. (35) is not rephasing invariant, this poses no problem when considering rephasing invariant quartets. With Eq. (35), one can obtain:

$$
\begin{aligned}
\operatorname{Im}( & \left.V_{i_{1} j_{1}} V_{i_{1} j_{2}}^{*} V_{i_{2} j_{2}} V_{i_{2} j_{1}}^{*}\right) \\
= & \sin 2 \theta\left\{4 s_{\theta}^{2} S_{i_{1} j_{1}} S_{i_{2} j_{2}} S_{i j} \epsilon_{i_{1} i_{2} i} \epsilon_{j_{1} j_{2} j}\right. \\
& +S_{i_{1} j_{1}} R_{i_{2} j_{1}} R_{i_{2} j_{2}} R_{i_{1} j_{2}}-R_{i_{1} j_{1}} S_{i_{2} j_{1}} R_{i_{2} j_{2}} R_{i_{1} j_{2}} \\
& \left.+R_{i_{1} j_{1}} R_{i_{2} j_{1}} S_{i_{2} j_{2}} R_{i_{1} j_{2}}-R_{i_{1} j_{1}} R_{i_{2} j_{1}} R_{i_{2} j_{2}} S_{i_{1} j_{2}}\right\} .
\end{aligned}
$$

Although Eq. (36) is not very illuminating, one can nevertheless illustrate that realistic values of $\left(V_{i_{1} j_{1}} V_{i_{1} j_{2}}^{*} V_{i_{2} j_{2}}\right.$ $\left.V_{i_{2} j_{1}}^{*}\right)$ can be obtained even in cases with less parametric freedom, as done in Sect. 3.3 below. The general case is analysed in Sect. 3.4. Before addressing those questions, we discuss two important aspects that deserve attention in the next two subsections: (i) the number of independent parameters and the most convenient choice for them, (ii) the fact that in this model, if tree level SFCNC were completely absent in one quark sector, then the CKM matrix would not be CP violating. One encounters again a deep connection [22] between the complexity of CKM and SFCNC, in the context of models with spontaneous $\mathrm{CP}$ violation.

\subsection{Parameters}

The CKM matrix $V$ requires 4 physical parameters, while the tree level SFCNC require 2 , since $\hat{r}_{[\mathrm{d}]}$ is a unit real vector. One can parametrise $\hat{r}_{[\mathrm{d}]}$ in terms of two angles $\theta_{d}, \varphi_{d}$ :

$\hat{r}_{[\mathrm{d}]}=\left(\sin \theta_{d} \cos \varphi_{d}, \sin \theta_{d} \sin \varphi_{d}, \cos \theta_{d}\right)$,

as shown in Fig. $1 .^{3}$ The orthogonal matrix $R$ requires 3 real parameters; together with $\theta$ and $\hat{r}_{[\mathrm{d}]}$, these 6 parameters match the parameters necessary to describe $V$ (4 parameters) and the products $\hat{r}_{[\mathrm{d}] j} \hat{r}_{[\mathrm{d}] k}, \hat{r}_{[\mathrm{u}] j} \hat{r}_{[\mathrm{u}] k}$ (2 parameters). However, in terms of $\mathcal{O}_{L}^{u}$ and $\mathcal{O}_{L}^{d}$, there are a priori $3+3$ real parameters;

\footnotetext{
${ }^{2}$ Here and in the following $c_{x} \equiv \cos x, s_{x} \equiv \sin x$.

3 The different products $\hat{r}_{[\mathrm{d}] i} \hat{r}_{[\mathrm{d}] j}$ controlling SFCNC are, simply, the areas of the shaded rectangular projections in the $(\hat{i}, \hat{j})$ planes in Fig. 1.
} 


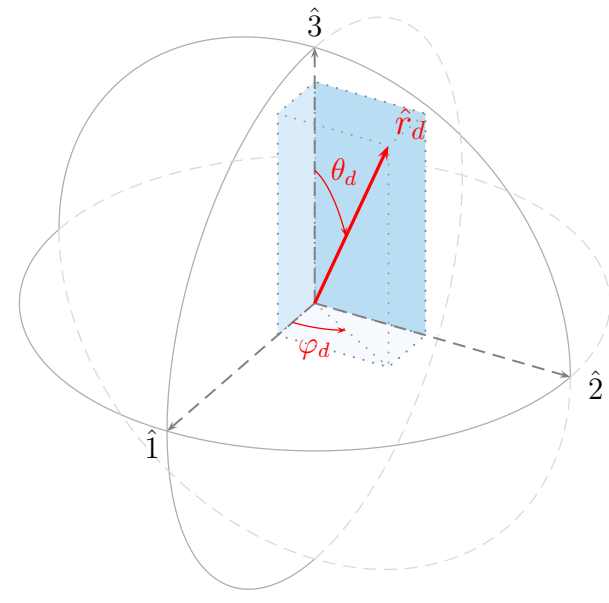

Fig. $1 \hat{r}_{[\mathrm{d}]}$

together with $\theta, 7$ parameters in all. This apparent mismatch can be readily understood: a common redefinition

$\mathcal{O}_{L}^{d} \mapsto\left(\begin{array}{ccc}\cos \alpha & \sin \alpha & 0 \\ -\sin \alpha & \cos \alpha & 0 \\ 0 & 0 & 1\end{array}\right) \mathcal{O}_{L}^{d}, \quad \mathcal{O}_{L}^{u} \mapsto\left(\begin{array}{ccc}\cos \alpha & \sin \alpha & 0 \\ -\sin \alpha & \cos \alpha & 0 \\ 0 & 0 & 1\end{array}\right) \mathcal{O}_{L}^{u}$,

leaves $R, \hat{r}_{[\mathrm{d}]}, \hat{r}_{[\mathrm{u}]}$ and $V$ unchanged, effectively removing one parameter from the $\mathcal{O}_{L}^{u}, \mathcal{O}_{L}^{d}$, parameter count. Consequently, it is convenient to adopt a parametrisation of $R$ of the form

$$
\begin{aligned}
R & =\left(\begin{array}{ccc}
1 & 0 & 0 \\
0 & c_{\alpha_{3}} & s_{\alpha_{3}} \\
0 & -s_{\alpha_{3}} & c_{\alpha_{3}}
\end{array}\right)\left(\begin{array}{ccc}
c_{\alpha_{2}} & 0 & s_{\alpha_{2}} \\
0 & 1 & 0 \\
-s_{\alpha_{2}} & 0 & c_{\alpha_{2}}
\end{array}\right)\left(\begin{array}{ccc}
c_{\alpha_{1}} & s_{\alpha_{1}} & 0 \\
-s_{\alpha_{1}} & c_{\alpha_{1}} & 0 \\
0 & 0 & 1
\end{array}\right) \\
& =\left(\begin{array}{ccc}
c_{\alpha_{1}} c_{\alpha_{2}} & s_{\alpha_{1}} c_{\alpha_{2}} & s_{\alpha_{2}} \\
-s_{\alpha_{1}} c_{\alpha_{3}}-c_{\alpha_{1}} s_{\alpha_{2}} s_{\alpha_{3}} & c_{\alpha_{1}} c_{\alpha_{3}}-s_{\alpha_{1}} s_{\alpha_{2}} s_{\alpha_{3}} & c_{\alpha_{2}} s_{\alpha_{3}} \\
s_{\alpha_{1}} s_{\alpha_{3}}-c_{\alpha_{1}} s_{\alpha_{2}} c_{\alpha_{3}} & -c_{\alpha_{1}} s_{\alpha_{3}}-s_{\alpha_{1}} s_{\alpha_{2}} c_{\alpha_{3}} & c_{\alpha_{2}} c_{\alpha_{3}}
\end{array}\right),
\end{aligned}
$$

and a parametrisation of $\mathcal{O}_{L}^{d}$ of the form ${ }^{4}$

$\mathcal{O}_{L}^{d}=\left(\begin{array}{ccc}c_{\alpha} & s_{\alpha} & 0 \\ -s_{\alpha} & c_{\alpha} & 0 \\ 0 & 0 & 1\end{array}\right)\left(\begin{array}{ccc}c_{\theta_{d}} c_{\varphi_{d}} & c_{\theta_{d}} s_{\varphi_{d}} & -s_{\theta_{d}} \\ -s_{\varphi_{d}} & c_{\varphi_{d}} & 0 \\ s_{\theta_{d}} c_{\varphi_{d}} & s_{\theta_{d}} s_{\varphi_{d}} & c_{\theta_{d}}\end{array}\right)$,

where $\hat{r}_{[\mathrm{d}]}$ is readily identified in the third row and the redundant $\alpha$, as in Eq. (38), can be set to $\alpha=0$. One can then concentrate on $\left\{\alpha_{1}, \alpha_{2}, \alpha_{3}, \theta_{d}, \varphi_{d}, \theta\right\}$ in order to reproduce a realistic CKM matrix.

\subsection{SFCNC and CP Violation in CKM}

In Eqs. (29)-(30), tree level SFCNC are a priori present in both the up and the down quark sectors and controlled by

4 The rows in Eq. (40) are simply unit vectors in spherical coordinates at $\mathbf{r}=\hat{r}_{[\mathrm{d}]}$, while $\alpha$, in Eq. (40) as in Eq. (38), produces an irrelevant rotation around $\hat{r}_{[\mathrm{d}]}$. $\hat{n}_{[\mathrm{q}] i}^{*} \hat{n}_{[\mathrm{q}] j}=\hat{r}_{[\mathrm{q}] i} \hat{r}_{[\mathrm{qq}] j}$. Therefore, if $\hat{r}_{[\mathrm{q}]}$ has a vanishing component, SFCNC in that sector $(q=u$ or $d)$ do only appear in one type of transition (the one not involving that component). If $\hat{r}_{[q]}$ had two vanishing components (then the remaining one equals \pm 1 ), there would not be SFCNC in that sector: interestingly, in this model, having no tree level SFCNC in one quark sector is incompatible with a CP violating CKM matrix. This can be readily checked by noticing that, in that case, in Eq. (34), the matrix with entries $S_{i j}$ has only a non vanishing row (column), corresponding to the absence of tree level SFCNC in the up (down) sector, for which $S_{i j}=R_{i j}$. Then, with $i_{1} \neq i_{2}$ and $j_{1} \neq j_{2}$, in Eq. (36) all terms except two out of the last four automatically vanish, and those two terms appear with opposite sign, giving $\operatorname{Im}\left(V_{i_{1} j_{1}} V_{i_{1} j_{2}}^{*} V_{i_{2} j_{2}} V_{i_{2} j_{1}}^{*}\right)=0$. As illustrated below, in Sect. 3.4, this implies that a lower bound on the size of the second largest component in $\hat{r}_{[q]}$ should exist, that is a lower bound on the intensity of some SFCNC in both quark sectors. Appendix A completes the discussion of the interplay in this model among flavour non-conservation and $\mathrm{CP}$ violation in the CKM matrix.

\subsection{A simple example}

As a simplified example of how a realistic CKM matrix can be obtained, consider a scenario with

$\hat{r}_{[\mathrm{d}]}=\left(\cos \varphi_{d}, \sin \varphi_{d}, 0\right)$.

Then, for $i_{1}=j_{1}=1, i_{2}=j_{2}=2$, Eq. (36) reduces to

$$
\begin{aligned}
& \operatorname{Im}\left(V_{11} V_{12}^{*} V_{22} V_{21}^{*}\right) \\
& \quad=\frac{1}{2}\left(R_{11} R_{21}+R_{12} R_{22}\right)\left(R_{12} R_{21}-R_{11} R_{22}\right) \sin 2 \varphi_{d} \sin 2 \theta
\end{aligned}
$$

Since the rows of $R$ form a complete orthonormal set of 3vectors,

$R_{11} R_{21}+R_{12} R_{22}=-R_{13} R_{23}, \quad R_{12} R_{21}-R_{11} R_{22}=-R_{33}$,

and Eq. (45) is further reduced to

$\operatorname{Im}\left(V_{11} V_{12}^{*} V_{22} V_{21}^{*}\right)=\frac{1}{2} R_{13} R_{23} R_{33} \sin 2 \varphi_{d} \sin 2 \theta$.

With $R$ in Eq. (39), Eq. (42) gives

$\operatorname{Im}\left(V_{11} V_{12}^{*} V_{22} V_{21}^{*}\right)=\frac{1}{8} \cos \alpha_{2} \sin 2 \alpha_{2} \sin 2 \alpha_{3} \sin 2 \varphi_{d} \sin 2 \theta$.

A complete example of this type which reproduces correctly the CKM matrix, is given by:

$\theta=\pi / 8$ 


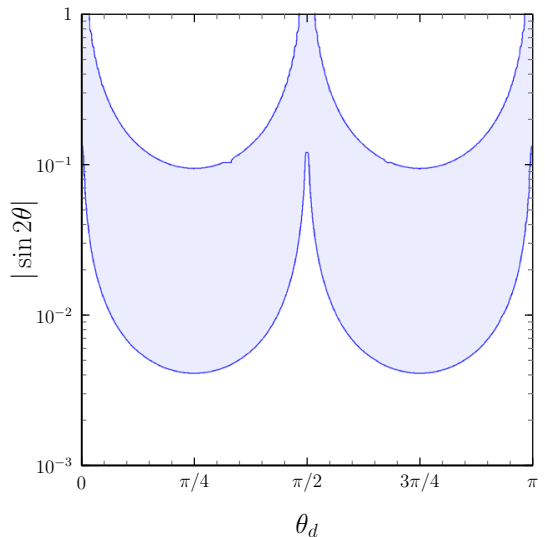

(a) $\sin 2 \theta$ vs. $\theta_{d}$

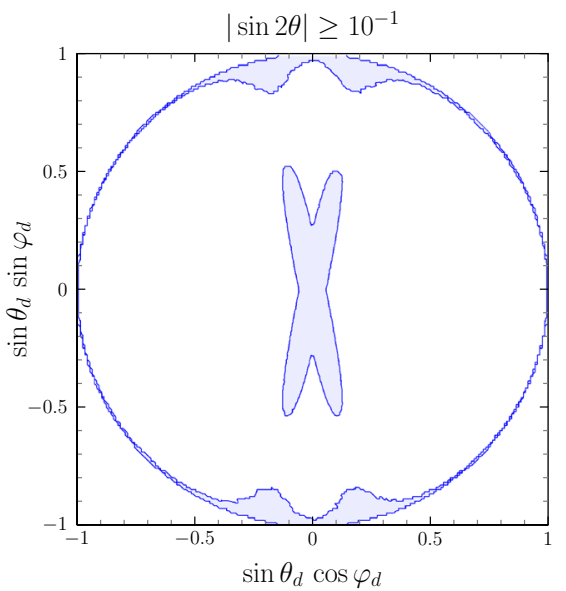

(d)

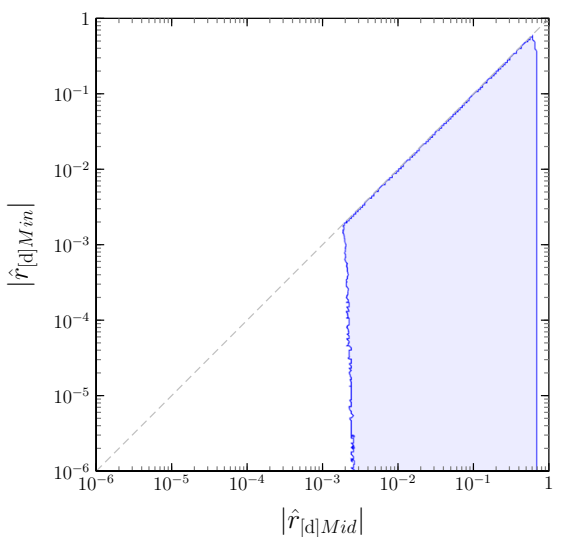

(b) $\left|\hat{r}_{[\mathrm{d}] M i n}\right|$ vs. $\left|\hat{r}_{[\mathrm{d}] M i d}\right|$.

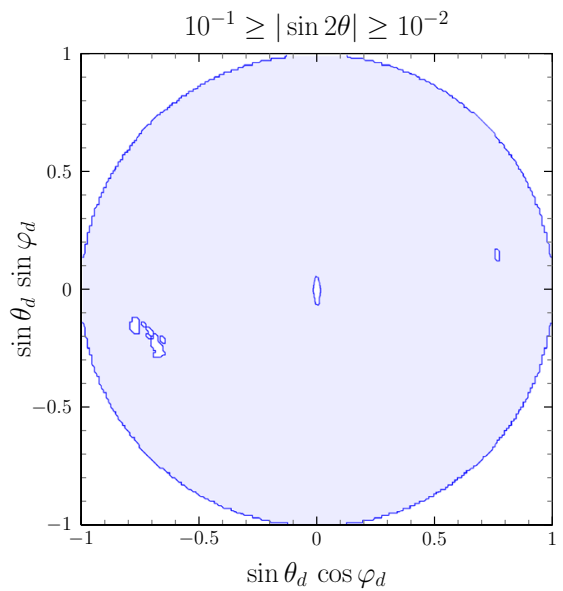

(e)

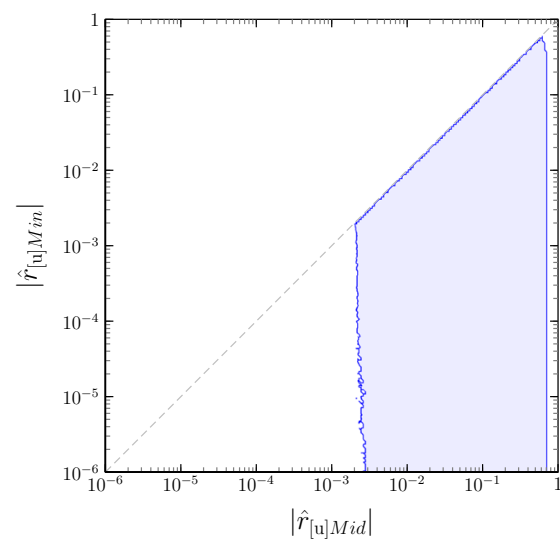

(c) $\left|\hat{r}_{[\mathrm{u}] M i n}\right|$ vs. $\left|\hat{r}_{[\mathrm{u}] M i d}\right|$.

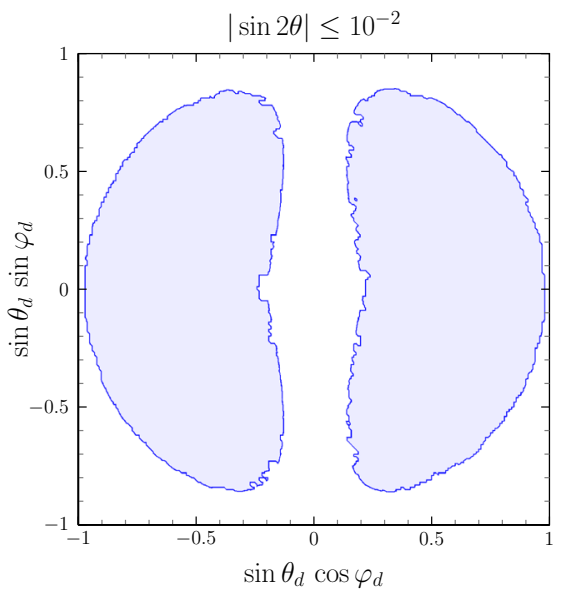

(f)

Fig. 2 Regions (at 99\% CL) which reproduce a realistic CKM matrix are shown

$R=\left(\begin{array}{ccc}1-7 \times 10^{-6} & 0 & -3.746 \times 10^{-3} \\ -1.536 \times 10^{-4} & -1+8.41 \times 10^{-4} & -0.041 \\ -3.743 \times 10^{-3} & 0.041 & -1+8.48 \times 10^{-4}\end{array}\right)$,

and

$\mathcal{O}_{L}^{d}=\left(\begin{array}{ccc}0 & 0 & -1 \\ 0.9509 & 0.3096 & 0 \\ 0.3096 & -0.9509 & 0\end{array}\right)$.

The parameters underlying Eqs. (47) and (48) have the values

$R: \quad \alpha_{1}=0, \alpha_{2}=-3.746 \times 10^{-3}, \alpha_{3}=0.041-\pi$,

$\mathcal{O}_{L}^{d}: \quad \theta_{d}=\pi / 2, \varphi_{d}=-1.2561, \alpha=0$.

For $R, \alpha_{1}=0$ has been chosen for simplicity, since in this scenario it does not enter Eq. (45). With the previous values, one can easily check that

$$
\begin{aligned}
& \left|V_{u s}\right|=0.2253,\left|V_{u b}\right|=3.75 \times 10^{-3} \\
& \left|V_{c b}\right|=0.041, \operatorname{Im}\left(V_{11} V_{12}^{*} V_{22} V_{21}^{*}\right)=3.195 \times 10^{-5} .
\end{aligned}
$$

Concerning the discussion in Sect. 3.2, this example shows that, although the complete absence of tree level FCNC in one sector is incompatible with a $\mathrm{CP}$ violating $\mathrm{CKM}$ matrix, this incompatibility does not extend to the case of tree level SFCNC circumscribed to only one type of transition (in this example, $d \leftrightarrow s$ transitions).

\subsection{General case}

The previous example illustrates that the CKM matrix can be adequately reproduced even in a restricted scenario where one has less number of free parameters. For the general case one can explore with a simple numerical analysis the regions of parameter space where the CKM matrix is in agreement with data, that is moduli $\left|V_{i j}\right|$ in the first two rows and the phase $\gamma$ agree with experimental results [23].

Figure $2 \mathrm{a}$ shows the region of the plane $|\sin 2 \theta|$ vs. $\theta_{d}$ which can yield a good CKM matrix. It is to be noticed that (i) regions rather close to $\theta=0, \pi / 2, \pi$, with $|\sin 2 \theta|<$ $10^{-2}$, are allowed and require $\theta_{d} \sim \pi / 4,3 \pi / 4$, while (ii) for $|\sin 2 \theta| \sim 1$, allowed regions require $\theta_{d} \sim 0, \pi / 2, \pi$. In any case, $\sin 2 \theta \neq 0$ is a necessary requirement, as expected, since there is no $\mathrm{CP}$ violation in that limit. 
Figure $2 \mathrm{~d}-\mathrm{f}$ also show the allowed regions in the $\sin \theta_{d}$ $\sin \varphi_{d}$ vs. $\sin \theta_{d} \cos \varphi_{d}$ plane, ${ }^{5}$ separating $|\sin 2 \theta| \geq 10^{-1}$ in Fig. $2 d, 10^{-1} \geq|\sin 2 \theta| \geq 10^{-2}$ in Fig. 2 e, and $10^{-2} \geq$ $|\sin 2 \theta|$ in Fig. $2 \mathrm{f}$.

Following the discussion in Sect. 3.2, one can sort the components of $\hat{r}_{[\mathrm{d}]}$ according to their size:

$\left|\hat{r}_{[\mathrm{d}] \operatorname{Min}}\right| \leq\left|\hat{r}_{[\mathrm{d}] \operatorname{Mid}}\right| \leq\left|\hat{r}_{[\mathrm{d}] \operatorname{Max}}\right|$

Incompatibility with a $\mathrm{CP}$ violating $\mathrm{CKM}$ matrix implies that $\left|\hat{r}_{[\mathrm{d}] \text { Mid }}\right| \neq 0$, while the simple example in Sect. 3.3 shows that $\left|\hat{r}_{[\mathrm{d}] \mathrm{Min}}\right|=0$ is allowed. Figure 2a shows the allowed region of $\left|\hat{r}_{[\mathrm{d}] M i n}\right|$ vs. $\left|\hat{r}_{[\mathrm{d}] M i d}\right|$ confirming that it is necessary that $\left|\hat{r}_{[\mathrm{d}] \text { Mid }}\right| \geq 2 \times 10^{-3}$, while Fig. 2 c corresponds similarly to $\hat{r}_{[\mathrm{u}]}$ : both figures illustrate that, in this model, necessarily, there is at least some minimal presence of tree SFCNC. ${ }^{6}$

In conclusion, it is clear that the first requirement on the model, i.e. that it can reproduce the observed CKM matrix, can be fulfilled.

\subsection{Strong CP}

It is well known that the solution to the $U(1)$ problem proposed by 't Hooft [24] leads to the so-called strong CP problem. In dealing with this problem the physical parameter (usually denoted $\bar{\theta}$ ), is given by

$\bar{\theta}=\theta_{\mathrm{QCD}}+\theta_{\mathrm{QFD}}$,

where $\theta_{\mathrm{QCD}}$ is the parameter which enters in the strong $\mathrm{CP}$ violating effective lagrangian

$\mathscr{L}_{\text {eff }} \propto \bar{\theta} \epsilon_{\mu \nu \alpha \beta} G_{a}^{\mu \nu} G_{a}^{\alpha \beta}$,

where $G_{a}^{\mu \nu}$ denotes the gluon field strength and $\theta_{\mathrm{QFD}}$ is given by

$\theta_{\mathrm{QFD}}=\arg \operatorname{det}\left(M_{u}^{0} M_{d}^{0}\right)$.

Experimentally, the upper limit on the neutron dipole moment implies $[25,26]$ that $\bar{\theta}$ is less than $\sim 10^{-10}$.

In the $\mathrm{SM} \bar{\theta}$ is an arbitrary dimensionless parameter, in principle of order one, with no justification for its smallness. This is the so-called strong CP problem. There is an elegant solution to this problem proposed by Peccei and Quinn $[27,28]$ which leads $[29,30]$ to the existence of an axion. The fact that axions have not been observed by experiment provides motivation to look for alternative solutions to the strong CP problem. One of them has been proposed $[31,32]$

\footnotetext{
5 That is, the projection on the $(\hat{1}, \hat{2})$ plane of the allowed regions in the surface of the sphere of Fig. 1.

${ }^{6}$ Notice that, consequently, $\theta_{d}=0, \pi$, are excluded: they give $\hat{r}_{[\mathrm{d}] \text { Min }}=\hat{r}_{[\mathrm{d}] \text { Mid }}=0, \hat{r}_{[\mathrm{d}] \text { Max }}= \pm 1$ and no SFCNC in the down sector; the resolution in Fig. $2 \mathrm{a}, \mathrm{d}$ is too coarse to observe that, while Fig. 2b, c clearly illustrate the point.
}

in the framework of models where $\mathrm{CP}$ is a good symmetry of the Lagrangian, only spontaneously broken. A specially interesting class of models are the ones based on the Barr-Nelson mechanism [33,34], with the minimal realization proposed in [35]. In this framework, $\theta_{\mathrm{QCD}}$ is put to zero, as a result of the $\mathrm{CP}$ invariance of the Lagrangian, and is calculable at higher orders in perturbation theory [36,37]. In the present model, $\theta_{\mathrm{QFD}}$ naturally vanishes at tree level, as can be seen from Eq. (13). It should be emphasized that the present model was constructed to solve the SFCNC problem and not the strong CP problem. The SFCNC problem generically arises in the $2 \mathrm{HDM}$ with spontaneous $\mathrm{CP}$ violation and no symmetry added to the Lagrangian, apart from gauge symmetry. In the proposed model, the SFCNC problem is solved through the introduction of a flavoured $\mathbb{Z}_{2}$ symmetry which leads to a physical $\mathrm{CP}$ violating vacuum phase that also generates a complex CKM matrix. Regarding the strong $\mathrm{CP}$ problem, as above mentioned, $\theta_{\mathrm{QFD}}$ vanishes at tree level in the present model. However, there is no additional natural suppression of $\theta_{\mathrm{QFD}}^{(1-\text { loop })}$ that ensures agreement with the experimental bound. Nevertheless, it is possible to find regions of parameter space where $\theta_{\mathrm{QFD}}^{(1-\text { loop })}$ is sufficiently suppressed. Of course, this implies some fine-tuning, but it should be stressed that the level of fine-tuning is much less severe that in the SM. In Appendix D we present an explicit evaluation $\theta_{\mathrm{QFD}}^{(1-\text { loop })}$ in the present model.

\section{The scalar potential with real couplings}

We consider the 2HDM with CP invariance and impose the $\mathbb{Z}_{2}$ symmetry of Eq. (2) which is only softly broken by a $\mu_{12}$ term. All couplings are real, so that CP holds at the Lagrangian level. The scalar potential can be written:

$$
\begin{aligned}
\mathscr{V}\left(\Phi_{1}, \Phi_{2}\right)= & \mu_{11}^{2} \Phi_{1}^{\dagger} \Phi_{1}+\mu_{22}^{2} \Phi_{2}^{\dagger} \Phi_{2}+\mu_{12}^{2}\left(\Phi_{1}^{\dagger} \Phi_{2}+\Phi_{2}^{\dagger} \Phi_{1}\right) \\
& +\lambda_{1}\left(\Phi_{1}^{\dagger} \Phi_{1}\right)^{2}+\lambda_{2}\left(\Phi_{2}^{\dagger} \Phi_{2}\right)^{2} \\
& +2 \lambda_{3}\left(\Phi_{1}^{\dagger} \Phi_{1}\right)\left(\Phi_{2}^{\dagger} \Phi_{2}\right)+2 \lambda_{4}\left(\Phi_{1}^{\dagger} \Phi_{2}\right)\left(\Phi_{2}^{\dagger} \Phi_{1}\right) \\
& +\lambda_{5}\left[\left(\Phi_{1}^{\dagger} \Phi_{2}\right)^{2}+\left(\Phi_{2}^{\dagger} \Phi_{1}\right)^{2}\right] .
\end{aligned}
$$

The vacuum expectation values are

$$
\left\langle\Phi_{1}\right\rangle=\left(\begin{array}{c}
0 \\
e^{i \theta_{1}} v_{1} / \sqrt{2}
\end{array}\right), \quad\left\langle\Phi_{2}\right\rangle=\left(\begin{array}{c}
0 \\
e^{i \theta_{2}} v_{2} / \sqrt{2}
\end{array}\right),
$$

and break electroweak symmetry spontaneously. As anticipated in Sect. 2, we use $\theta=\theta_{2}-\theta_{1}, v^{2}=v_{1}^{2}+v_{2}^{2}$, $c_{\beta}=\cos \beta \equiv v_{1} / v, s_{\beta}=\sin \beta \equiv v_{2} / v$ and $t_{\beta} \equiv \tan \beta$, with $v_{1} \geq 0, v_{2} \geq 0$. 


\subsection{Minimization}

The minimization conditions for $V\left(v_{1}, v_{2}, \theta\right) \equiv \mathscr{V}\left(\left\langle\Phi_{1}\right\rangle\right.$, $\left.\left\langle\Phi_{2}\right\rangle\right)$ are

$$
\begin{aligned}
\frac{\partial V}{\partial \theta}= & -v_{1} v_{2} \sin \theta\left(\mu_{12}^{2}+2 \lambda_{5} v_{1} v_{2} \cos \theta\right)=0 \\
\frac{\partial V}{\partial v_{1}}= & \mu_{11}^{2} v_{1}+\lambda_{1} v_{1}^{3}+\left(\lambda_{3}+\lambda_{4}\right) v_{1} v_{2}^{2} \\
& +v_{2}\left(\mu_{12}^{2} \cos \theta+\lambda_{5} v_{1} v_{2} \cos 2 \theta\right)=0 \\
\frac{\partial V}{\partial v_{2}}= & \mu_{22}^{2} v_{2}+\lambda_{2} v_{2}^{3}+\left(\lambda_{3}+\lambda_{4}\right) v_{1}^{2} v_{2} \\
& +v_{1}\left(\mu_{12}^{2} \cos \theta+\lambda_{5} v_{1} v_{2} \cos 2 \theta\right)=0
\end{aligned}
$$

In order to have spontaneous $\mathrm{CP}$ violation, we consider a solution $\left\{v_{1}, v_{2}, \theta\right\}$ of Eqs. (57)-(59) with $\theta \neq 0, \pm \pi / 2, \pm \pi$. From Eq. (57) one obtains

$$
\cos \theta=\frac{-\mu_{12}^{2}}{2 \lambda_{5} v_{1} v_{2}} .
$$

Notice that, in addition to $\theta,-\theta$ is also a solution. It is obvious that for $\theta=0, \pi$ the vacuum is $\mathrm{CP}$ invariant. It has also been shown [13] that for $\theta=\pi / 2$ the vacuum is also $\mathrm{CP}$ invariant. Note from Eq. (60) that $\theta= \pm \pi / 2$ is obtained when $\mu_{12}^{2}=0$. In this case, the scalar potential is invariant under the $\mathbb{Z}_{2}$ symmetry of Eq. (2). This symmetry allows the two scalar fields $\Phi_{1}, \Phi_{2}$ to have either equal or opposite CP parities. It is this freedom that is used to construct a simple proof [13] that for $\theta= \pm \pi / 2$, the vacuum is CP invariant.

One can trade $\mu_{11}^{2}, \mu_{22}^{2}$ and $\mu_{12}^{2}$ for other parameters using Eqs. (57)-(59):

$\mu_{12}^{2}=-2 \lambda_{5} v_{1} v_{2} \cos \theta$,

$\mu_{11}^{2}=-\left(\lambda_{1} v_{1}^{2}+\left(\lambda_{3}+\lambda_{4}-\lambda_{5}\right) v_{2}^{2}\right)$,

$\mu_{22}^{2}=-\left(\lambda_{2} v_{2}^{2}+\left(\lambda_{3}+\lambda_{4}-\lambda_{5}\right) v_{1}^{2}\right)$.

That is, imposing Eqs. (61)-(63) on $\mathscr{V}\left(\Phi_{1}, \Phi_{2}\right)$ in Eq. (55), one is selecting a scalar potential where, at least, the necessary minimization conditions in Eqs. (57)-(59) are satisfied for generic $\left\{v_{1}, v_{2}, \theta\right\}$. One can in addition choose $v_{1}^{2}+v_{2}^{2}=$ $v^{2}=(246 \mathrm{GeV})^{2}$ for appropriate electroweak symmetry breaking without loss of generality (this is enforced, for example, by a simple rescaling of the parameters in the potential). Fixing $v^{2}$ in that manner, one is left with a candidate minimum characterised by the values of $\theta$ and $\tan \beta=v_{2} / v_{1}$, which remain free parameters that we can choose at will, up to the different constraints on the scalar potential to be discussed later:

1. the potential is bounded from below and $V\left(v_{1}, v_{2}, \theta\right)$ is the lowest lying minimum;
2. perturbative unitarity bounds on scattering processes in the scalar sector are respected.

Expanding $\Phi_{j}$ around the candidate vacuum in Eq. (56)

$\Phi_{j}=e^{i \theta_{j}}\left(\begin{array}{c}\varphi_{j}^{+} \\ \frac{1}{\sqrt{2}}\left(v_{j}+\rho_{j}+i \eta_{j}\right)\end{array}\right)$,

we can now explore the different mass terms for the charged and neutral scalars. Requiring that the mass parameters of all the physical scalars are positive ensures, at least, that the candidate minimum is a local minimum of the potential. In the Higgs basis of Eq. (5), the expansion of the fields reads

$$
\begin{gathered}
H_{1}=\left(\begin{array}{c}
G^{+} \\
\left(v+H^{0}+i G^{0}\right) / \sqrt{2}
\end{array}\right), \quad H_{2}=\left(\begin{array}{c}
\mathrm{H}^{+} \\
\left(R^{0}+i I^{0}\right) / \sqrt{2}
\end{array}\right), \\
\left(\begin{array}{c}
G^{+} \\
\mathrm{H}^{+}
\end{array}\right)=\mathcal{R}_{\beta}\left(\begin{array}{c}
\varphi_{1}^{+} \\
\varphi_{2}^{+}
\end{array}\right),\left(\begin{array}{c}
G^{0} \\
I^{0}
\end{array}\right)=\mathcal{R}_{\beta}\left(\begin{array}{l}
\eta_{1} \\
\eta_{2}
\end{array}\right),\left(\begin{array}{c}
H^{0} \\
R^{0}
\end{array}\right)=\mathcal{R}_{\beta}\left(\begin{array}{c}
\rho_{1} \\
\rho_{2}
\end{array}\right),
\end{gathered}
$$

with the would-be Goldstone bosons $G^{ \pm}$and $G^{0}$ readily identified

$G^{ \pm}=c_{\beta} \varphi_{1}^{ \pm}-s_{\beta} \varphi_{2}^{ \pm}, \quad G^{0}=c_{\beta} \eta_{1}-s_{\beta} \eta_{2}$.

\subsection{Scalar masses and mixings}

\subsubsection{Charged scalar}

The transformation into the Higgs basis also gives the mass term of the charged scalar $\mathrm{H}^{ \pm}=s_{\beta} \varphi_{1}^{ \pm}-c_{\beta} \varphi_{2}^{ \pm}$,

$\mathscr{V}\left(\Phi_{1}, \Phi_{2}\right) \supset v^{2}\left(\lambda_{5}-\lambda_{4}\right) \mathrm{H}^{+} \mathrm{H}^{-} \Rightarrow m_{\mathrm{H}^{ \pm}}^{2}=v^{2}\left(\lambda_{5}-\lambda_{4}\right)$.

Notice that, in order to choose a set of independent parameters, Eq. (68) will allow us to trade $\lambda_{4}$ for $m_{\mathrm{H}^{ \pm}}^{2}$ and $\lambda_{5}$. Furthermore, since $\lambda_{5}$ and $\lambda_{4}$ are subject to the constraints on the scalar potential discussed in Appendix $\mathrm{B}, m_{\mathrm{H}^{ \pm}}$has a limited allowed range: for example, if $\lambda_{5}-\lambda_{4}<20$, then it follows that $m_{\mathrm{H}^{ \pm}}<9 m_{\mathrm{h}}$.

\subsubsection{Neutral scalars}

For the neutral scalar sector, the mass terms are

$$
\mathscr{V}\left(\Phi_{1}, \Phi_{2}\right) \supset \frac{1}{2}\left(\begin{array}{lll}
H^{0} & R^{0} & I^{0}
\end{array}\right) \mathcal{M}_{0}^{2}\left(\begin{array}{c}
H^{0} \\
R^{0} \\
I^{0}
\end{array}\right)
$$

with $\mathcal{M}_{0}^{2}=\mathcal{M}_{0}^{2 T}$, and

$$
\begin{aligned}
& {\left[\mathcal{M}_{0}^{2}\right]_{11}=2 v^{2}\left\{\lambda_{1} c_{\beta}^{4}+\lambda_{2} s_{\beta}^{4}+2 c_{\beta}^{2} s_{\beta}^{2}\left[\lambda_{345}+2 \lambda_{5} c_{\theta}^{2}\right]\right\},} \\
& {\left[\mathcal{M}_{0}^{2}\right]_{22}=2 v^{2}\left\{c_{\beta}^{2} s_{\beta}^{2}\left(\lambda_{1}+\lambda_{2}-2 \lambda_{345}\right)+\lambda_{5}\left(c_{\beta}^{2}-s_{\beta}^{2}\right)^{2} c_{\theta}^{2}\right\},}
\end{aligned}
$$




$$
\begin{aligned}
& {\left[\mathcal{M}_{0}^{2}\right]_{12}=2 v^{2} s_{\beta} c_{\beta}\left\{-\lambda_{1} c_{\beta}^{2}+\lambda_{2} s_{\beta}^{2}\right.} \\
& \left.\left.\qquad+\left(c_{\beta}^{2}-s_{\beta}^{2}\right)\left[\lambda_{345}+2 \lambda_{5} c_{\theta}^{2}\right]\right)\right\} \\
& {\left[\mathcal{M}_{0}^{2}\right]_{13}=-v^{2} \lambda_{5} s_{2 \beta} s_{2 \theta},} \\
& {\left[\mathcal{M}_{0}^{2}\right]_{23}=-v^{2} \lambda_{5} c_{2 \beta} s_{2 \theta},} \\
& {\left[\mathcal{M}_{0}^{2}\right]_{33}=2 v^{2} \lambda_{5} s_{\theta}^{2},}
\end{aligned}
$$

with, we recall, the shorthand notation $c_{x}=\cos x, s_{x}=$ $\sin x$, and $\lambda_{345} \equiv \lambda_{3}+\lambda_{4}-\lambda_{5}$.

For $\lambda_{5} s_{2 \theta} \neq 0$, attending to $\left[\mathcal{M}_{0}^{2}\right]_{13} \neq 0$ and $\left[\mathcal{M}_{0}^{2}\right]_{23} \neq 0$ above, there is scalar-pseudoscalar mixing, as it is expected from spontaneous breaking of CP in the scalar sector. $\mathcal{M}_{0}^{2}$ is diagonalised through a real orthogonal transformation $\mathcal{R}$

$\mathcal{R}^{T} \mathcal{M}_{0}^{2} \mathcal{R}=\operatorname{diag}\left(m_{\mathrm{h}}^{2}, m_{\mathrm{H}}^{2}, m_{\mathrm{A}}^{2}\right), \quad \mathcal{R}^{-1}=\mathcal{R}^{T}$.

The physical neutral scalars are

$$
\left(\begin{array}{l}
\mathrm{h} \\
\mathrm{H} \\
\mathrm{A}
\end{array}\right)=\mathcal{R}^{T}\left(\begin{array}{c}
H^{0} \\
R^{0} \\
I^{0}
\end{array}\right),
$$

and we assume $h$ to be the lightest one, the Higgs-like neutral scalar with $m_{\mathrm{h}}=125 \mathrm{GeV}$. With $\mathcal{M}_{0}^{2}$ in Eq. (70), $\mathcal{R}$ "mixes", a priori, all three neutral scalars. It is interesting to notice that

$\operatorname{Tr}\left[\mathcal{M}_{0}^{2}\right]=m_{\mathrm{h}}^{2}+m_{\mathrm{H}}^{2}+m_{\mathrm{A}}^{2}=2 v^{2}\left[\lambda_{1} c_{\beta}^{2}+\lambda_{2} s_{\beta}^{2}+\lambda_{5}\right]$,

and

$$
\operatorname{det}\left[\mathcal{M}_{0}^{2}\right]=m_{\mathrm{h}}^{2} m_{\mathrm{H}}^{2} m_{\mathrm{A}}^{2}=2 v^{6} \lambda_{5}\left(\lambda_{1} \lambda_{2}-\lambda_{345}^{2}\right) \sin ^{2} 2 \beta \sin ^{2} \theta
$$

As explained in Appendix B, since it is necessary that $\lambda_{5}>0$, it is also required that $\lambda_{1} \lambda_{2}>\lambda_{345}^{2}$ for $V\left(v_{1}, v_{2}, \theta\right)$ to be, at least, a local minimum (for which, necessarily, $\operatorname{det}\left[\mathcal{M}_{0}^{2}\right]>$ $0)$.

Equations (73) and (74) encode in a transparent manner several interesting properties of the model. First, since the different $\lambda_{j}$ are bounded by the requirements discussed in Appendix B (in particular by perturbative unitarity), and $\sin ^{2} 2 \beta \leq 1$ and $\sin ^{2} \theta \leq 1$, the masses of the new scalars $\mathrm{H}, \mathrm{A}, \mathrm{H}^{ \pm}$, have a limited allowed range. For a very crude estimate, consider for example $\lambda_{1} c_{\beta}^{2}+\lambda_{2} s_{\beta}^{2}+\lambda_{5} \sim 10$ in Eq. (73): with $v \simeq 2 m_{\mathrm{h}}, m_{\mathrm{H}}^{2}+m_{\mathrm{A}}^{2} \sim 80 m_{\mathrm{h}}^{2}$ and it is clear that the smaller among $m_{\mathrm{H}}$ and $m_{\mathrm{A}}$ cannot be larger than $\sim 6 m_{\mathrm{h}}$, while the larger among $m_{\mathrm{H}}$ and $m_{\mathrm{A}}$ cannot be larger than $\sim 9 m_{\mathrm{h}}$.

On the other hand, from Eq. (74), for $\sin 2 \beta \ll 1$, at least one neutral scalar should be light and either $\tan \beta \gg 1$ or $\tan ^{-1} \beta \gg 1$, which enhance SFCNC couplings. One can than expect that $\sin 2 \beta \ll 1$ will be disfavoured by the constraints discussed in Sect. 6, while $\tan \beta \sim \tan ^{-1} \beta \sim 1$ are easier to accommodate. Finally, it is to be noticed that for $\sin \theta=1$, there is no mixing among $\{\mathrm{h}, \mathrm{H}\}$ and $\mathrm{A}$ (and
$m_{\mathrm{A}}^{2}=2 \lambda_{5} v^{2}$ ), and, as discussed, no spontaneous CP violation and a real CKM matrix. For $\sin \theta=0, m_{\mathrm{A}}=0$ and thus for $|\sin \theta| \ll 1$, one could expect again the presence of at least one light scalar.

From the previous comments, it emerges that in this model there is limited room to have a scalar sector where (i) $\mathrm{h}$ is a Higgs boson with quite SM-like properties and (ii) $m_{\mathrm{H}^{ \pm}}$, $m_{\mathrm{H}}, m_{\mathrm{A}} \gg m_{\mathrm{h}}$. In this model, there is no decoupling regime for the new scalars. It is also clear, with these values, that the new scalars should be produced at the LHC. Nevertheless, the most relevant production and decay modes for their discovery will vary significantly between different regions of parameter space, including the Yukawa couplings discussed in Sect. 5, and also the details of the lepton sector, and are thus beyond the scope of this work.

\subsection{A simple analysis of the scalar sector}

As a first step in the direction of the complete analysis of Sect. 6, in this subsection we analyse the available parameter space of the scalar sector of the model, considering the following constraints.

- Agreement with electroweak precision data, in particular the oblique parameters $S$ and $T$ [38].

- Boundedness of the scalar potential and perturbative unitarity of the scattering processes, controlled by the scalar quartic couplings $\lambda_{j}$, as described, respectively, in Appendices B.2 and B.3.

- We only consider $m_{\mathrm{H}^{ \pm}}, m_{\mathrm{H}}, m_{\mathrm{A}} \geq 150 \mathrm{GeV}$; although masses of new scalars below $150 \mathrm{GeV}$ are not automatically excluded by existing constraints, they would require specific analyses, interesting on their own, which are out of the scope of the present work. Furthermore, attending to Eq. (74) and the related discussion, imposing this requirement on $m_{\mathrm{H}}$ and $m_{\mathrm{A}}$ translates into a lower bound on $s_{2 \beta}^{2}$ and $s_{\theta}^{2}$. For a simple estimate one can take $\lambda_{5}\left(\lambda_{1} \lambda_{2}-\lambda_{345}^{2}\right)<10^{2}$, which gives (for $m_{\mathrm{H}}$, $\left.m_{\mathrm{A}} \geq 150 \mathrm{GeV}\right) s_{2 \beta}^{2}, s_{\theta}^{2}>10^{-4}$. In terms of $t_{\beta}$, this means $10^{-2}<t_{\beta}<10^{2}$. On the contrary, since the quantity relevant for the obtention of a realistic CKM matrix is $\sin 2 \theta$ rather than $\sin \theta, s_{\theta}^{2}>10^{-4}$ is only relevant for $\theta \sim 0, \pi$, while $|\sin 2 \theta| \ll 1$ with $\theta \sim \frac{\pi}{2}, \frac{3 \pi}{2}$ is allowed.

- The analyses of Higgs signal strengths from the ATLAS and CMS collaborations, e.g. [39], put constraints on different couplings of h. Overall, the resulting picture corresponds to an h which is quite SM-like. For that reason, in order to discard from this simple analysis the regions of parameter space that these constraints will in any case eliminate in the complete analysis of Sect. 6, we require here $\left|\mathcal{R}_{11}\right| \geq 0.9$. 
Fig. 3 Regions allowed at 99\% CL by the requirements on the scalar sector

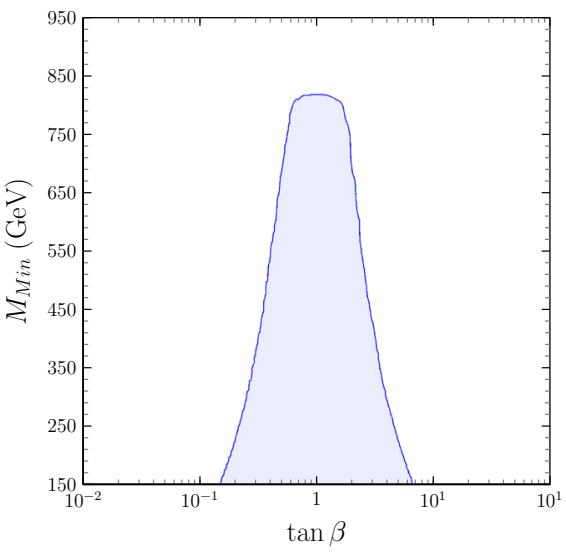

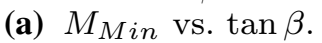

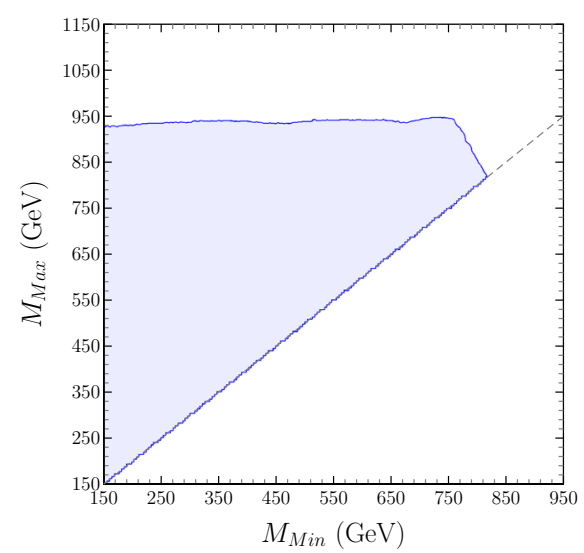

(c) $M_{\text {Max }}$ vs. $M_{M i n}$.

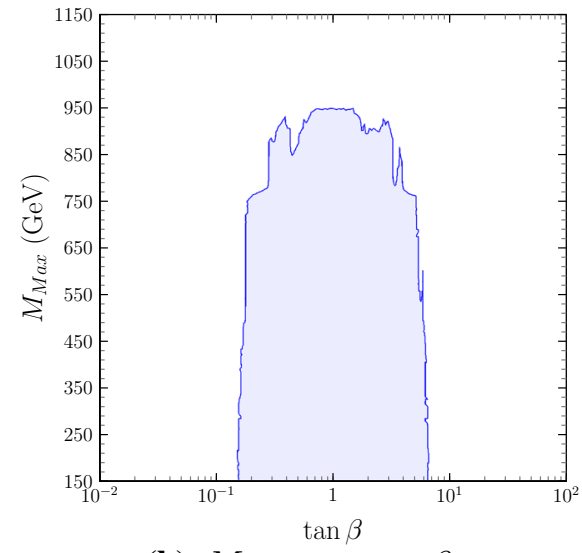

(b) $M_{\text {Max }}$ vs. $\tan \beta$.

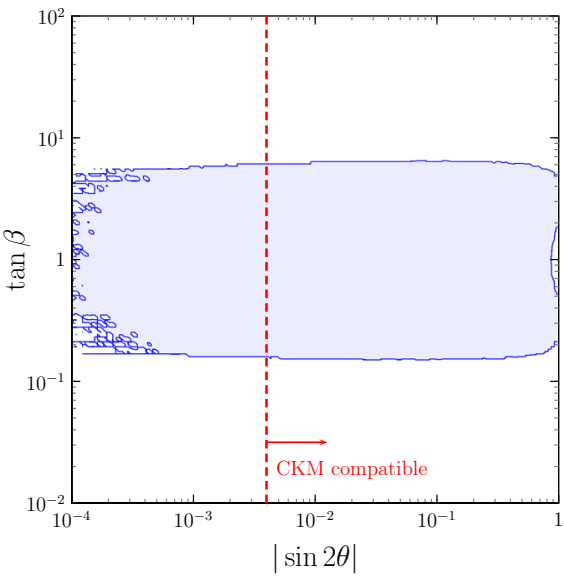

(d) $\tan \beta$ vs. $|\sin 2 \theta|$.
Although the analysis of Sect. 3.4 already sets a lower bound $|\sin 2 \theta| \geq 4 \times 10^{-3}$ in order to obtain the correct CKM matrix, we do not impose it here (it corresponds to the dashed vertical line in Fig. 3d). A detailed discussion of one convenient parametrisation of all quantities related to the scalar sector is given in Appendix B.1.

With these ingredients, the allowed regions in Figs. 3 and 4 are obtained. We introduce

$M_{M i n} \equiv \min \left(m_{\mathrm{H}}, m_{\mathrm{A}}, m_{\mathrm{H}^{ \pm}}\right), \quad M_{\text {Max }} \equiv \max \left(m_{\mathrm{H}}, m_{\mathrm{A}}, m_{\mathrm{H}^{ \pm}}\right)$

Figure 3 a shows that, with the simple requirements enumerated above, all new scalars cannot have, simultaneously, masses above $\sim 750 \mathrm{GeV}$. These values are in rough agreement with the previous naive estimates. Figure $3 \mathrm{~b}$ shows in addition that no new scalar can be heavier than $\sim 950 \mathrm{GeV}$. It is also clear that the largest values of the scalar masses correspond to $t_{\beta} \simeq 1$, while only a reduced range of values of $t_{\beta}$ is allowed, $10^{-1}<t_{\beta}<10$. Figure $3 \mathrm{c}$ shows that the limitations on allowed $M_{M i n}$ and $M_{M a x}$ appear to be rather independent: for example, $M_{\text {Max }} \sim 850 \mathrm{GeV}$ is compatible with any value of $M_{M i n}$ below $750 \mathrm{GeV}$.

Figure $4 \mathrm{a}, \mathrm{b}$ illustrate that any ordering of the masses $m_{\mathrm{H}}$, $m_{\mathrm{A}}, m_{\mathrm{H}^{ \pm}}$is allowed, and no particular restriction arises.
Having introduced the physical scalars and analysed some relevant aspects of the scalar sector, we can now turn back to $\mathscr{L}_{\mathrm{Y}}$ in Eq. (7) and discuss the Yukawa couplings of the physical quarks and scalars.

\section{Physical Yukawa couplings}

The Yukawa lagrangian in Eq. (7)

$\mathscr{L}_{\mathrm{Y}}=\mathscr{L}_{\overline{\mathrm{q} q}}+\mathscr{L}_{G \bar{q} \mathrm{q}}+\mathscr{L}_{\mathrm{h} \overline{\mathrm{q}}}+\mathscr{L}_{\mathrm{H} \overline{\mathrm{q}} \mathrm{q}}+\mathscr{L}_{\mathrm{A} \overline{\mathrm{q}}}+\mathscr{L}_{\mathrm{H}^{ \pm} \overline{\mathrm{q}}}$,

gives the mass terms for quarks $\mathscr{L}_{\overline{\mathrm{q} q}}=-\bar{d}_{L} M_{d} d_{R}-$ $\bar{u}_{L} M_{u} u_{R}+$ H.c, the couplings to the would-be Goldstone bosons $\mathscr{L}_{G \bar{q} \mathrm{q}}$,

$$
\begin{aligned}
\mathscr{L}_{G \overline{\mathrm{q} q}}= & -i \frac{G^{0}}{v}\left[\bar{d} \gamma_{5} d-\bar{u} \gamma_{5} u\right] \\
& -\frac{\sqrt{2} G^{+}}{v}\left[\bar{u}_{L} V M_{d} d_{R}-\bar{u}_{R} M_{u} V d_{L}\right] \\
& -\frac{\sqrt{2} G^{-}}{v}\left[\bar{d}_{R} M_{d} V^{\dagger} u_{L}-\bar{d}_{L} V^{\dagger} M_{u} u_{R}\right],
\end{aligned}
$$




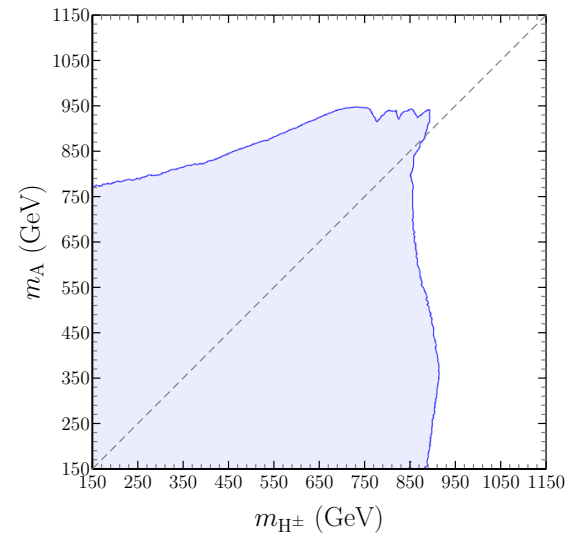

(a) $m_{\mathrm{A}}$ vs. $m_{\mathrm{H}}$.

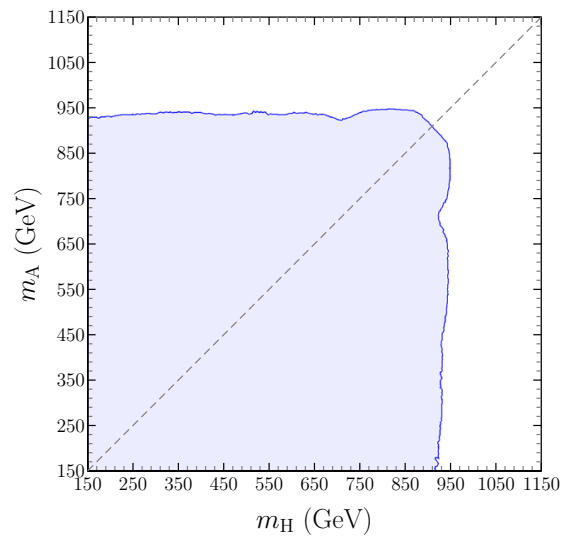

(b) $m_{\mathrm{A}}$ vs. $m_{\mathrm{H}}$.

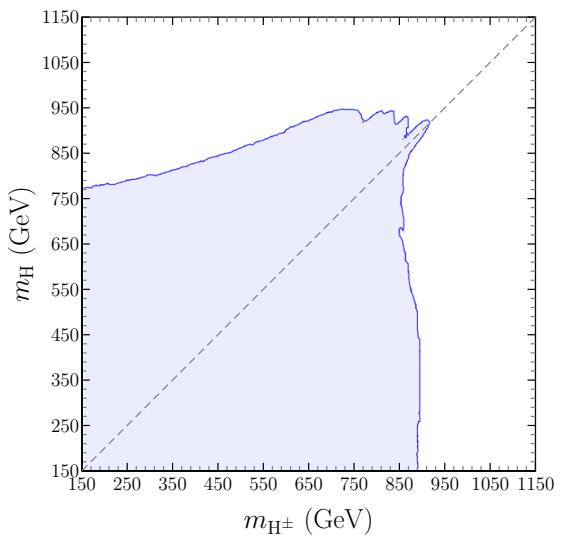

(c) $m_{\mathrm{H}}$ vs. $m_{\mathrm{H}^{ \pm}}$.

Fig. 4 Regions allowed at 99\% CL by the requirements on the scalar sector

and the Yukawa couplings to the neutral and charged scalars $\mathscr{L}_{\text {S̄̄q }}, S=\mathrm{h}, \mathrm{H}, \mathrm{A}, \mathrm{H}^{ \pm}$. Introducing the hermitian and antihermitian combinations

$H_{q} \equiv \frac{N_{q}+N_{q}^{\dagger}}{2}, \quad A_{q} \equiv \frac{N_{q}-N_{q}^{\dagger}}{2}$,

we have

$$
\begin{aligned}
\mathscr{L}_{S \overline{\mathrm{q}} \mathrm{q}}= & -\frac{S}{v}\left\{\bar{d}\left[\mathcal{R}_{1 s} M_{d}+\mathcal{R}_{2 s} H_{d}+i \mathcal{R}_{3 s} A_{d}\right] d\right. \\
& \left.+\bar{d}\left[\mathcal{R}_{2 s} A_{d}+i \mathcal{R}_{3 s} H_{d}\right] \gamma_{5} d\right\} \\
& -\frac{S}{v}\left\{\bar{u}\left[\mathcal{R}_{1 s} M_{u}+\mathcal{R}_{2 s} H_{u}-i \mathcal{R}_{3 s} A_{u}\right] u\right. \\
& \left.+\bar{u}\left[\mathcal{R}_{2 s} A_{u}-i \mathcal{R}_{3 s} H_{u}\right] \gamma_{5} u\right\},
\end{aligned}
$$

with $s=1,2,3$ for $S=\mathrm{h}, \mathrm{H}$, A, respectively, and

$$
\begin{aligned}
\mathscr{L}_{\mathrm{H}^{ \pm} \overline{\mathrm{q}}}= & -\frac{\sqrt{2} \mathrm{H}^{+}}{v}\left[\bar{u}_{L} V N_{d} d_{R}-\bar{u}_{R} N_{u}^{\dagger} V d_{L}\right] \\
& -\frac{\sqrt{2} \mathrm{H}^{-}}{v}\left[\bar{d}_{R} N_{d}^{\dagger} V^{\dagger} u_{L}-\bar{d}_{L} V^{\dagger} N_{u} u_{R}\right] .
\end{aligned}
$$

With Eqs. (29) and (30), $\left[H_{q}\right]_{i j}$ and $\left[A_{q}\right]_{i j}$ in Eq. (78) read

$$
\begin{aligned}
& {\left[H_{q}\right]_{i j}=t_{\beta} \delta_{i j} m_{q_{i}}-\left(t_{\beta}+t_{\beta}^{-1}\right) \hat{n}_{[\mathrm{q}] i}^{*} \hat{n}_{[\mathrm{qq}] j} \frac{m_{d_{i}}+m_{d_{j}}}{2},} \\
& {\left[A_{q}\right]_{i j}=\left(t_{\beta}+t_{\beta}^{-1}\right) \hat{n}_{[\mathrm{q}] i}^{*} \hat{n}_{[\mathrm{q}] j} \frac{m_{d_{i}}-m_{d_{j}}}{2} .}
\end{aligned}
$$

We recall - see for example [40] - that, for flavour changing Yukawa couplings of quarks $q_{j}, q_{k}$ and a scalar $S$, of the form $\mathscr{L}_{\mathrm{S}_{\mathrm{j} \mathrm{q}_{\mathrm{k}}}}=-S \bar{q}_{j}\left(a_{j k}+i b_{j k} \gamma_{5}\right) q_{k}+$ H.c $, \quad a_{j k}, b_{j k} \in \mathbb{C}$,

$\mathrm{CP}$ conservation requires $\operatorname{Re}\left(a_{j k}^{*} b_{j k}\right)=0$. In this model

$$
\operatorname{Re}\left(a_{j k}^{*} b_{j k}\right) \propto \mathcal{R}_{2 s} \mathcal{R}_{3 s}\left(t_{\beta}+t_{\beta}^{-1}\right)^{2} m_{q_{j}} m_{q_{k}}\left|\hat{n}_{[\mathrm{q}] j}^{*} \hat{n}_{[\mathrm{q}] k}\right|^{2},
$$

and thus, with $\mathcal{R}$ mixing all three neutral scalars, the flavour changing Yukawa couplings are CP violating. For the charged scalar, we have

$\operatorname{Re}\left(a_{j k}^{*} b_{j k}\right) \propto \operatorname{Im}\left(\left(V N_{d}\right)_{j k}^{*}\left(N_{u}^{\dagger} V\right)_{j k}\right)$,

and thus in general, even for real $N_{q}$, the Yukawa couplings of $\mathrm{H}^{ \pm}$are also $\mathrm{CP}$ violating.

For flavour conserving Yukawa couplings

$\mathscr{L}_{\text {Sqq }}=-S \bar{q}\left(a+i b \gamma_{5}\right) q, \quad a, b \in \mathbb{R}$,

$\mathrm{CP}$ conservation requires $a b=0$. Then, for the coupling of the neutral scalar $S$, with Eqs. (81) and (82), we have

$$
\begin{aligned}
& a b \propto \mathcal{R}_{3 s} m_{q_{j}}^{2}\left(\mathcal{R}_{1 s}+\mathcal{R}_{2 s}\left[t_{\beta}-\left(t_{\beta}+t_{\beta}^{-1}\right)\left|\hat{n}_{[\mathrm{q}] j}\right|^{2}\right]\right) \\
& \quad\left(t_{\beta}-\left(t_{\beta}+t_{\beta}^{-1}\right)\left|\hat{n}_{[\mathrm{q}] j}\right|^{2}\right),
\end{aligned}
$$

and thus the flavour conserving Yukawa couplings violate $\mathrm{CP}$ as long as the mixing in the scalar sector connects A with $\mathrm{h}$, $\mathrm{H}$. Contributions to the electric dipole moment of the neutron arise from Eq. (87), but the suppression due to the $m_{q_{j}}^{2}$ factor for $q_{j}=u, d$, together with the need of different non-zero mixings in the scalar sector, keep them within experimental bounds [41].

\section{Phenomenology}

\subsection{Analysis and constraints}

In Sect. 3 we have shown that the model can give a CKM mixing matrix in agreement with data. We have also explored some aspects of the scalar sector in Sect. 4. In this section we analyse the model considering simultaneously (i) obtention of an adequate CKM matrix (moduli $\left|V_{i j}\right|$ in the first and second rows and phase $\gamma$ in agreement with data), (ii) a scalar 

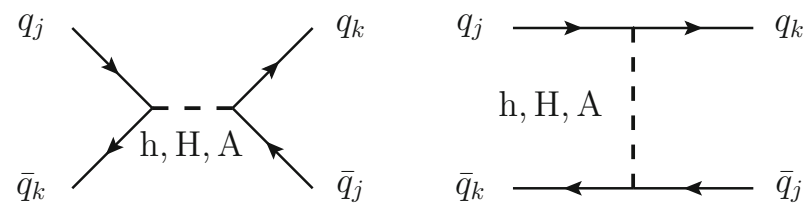

(a)

Fig. 5 Diagrams contributing to: a meson mixing, $\mathbf{b} b \rightarrow s \gamma$

sector verifying boundedness, perturbative unitarity, oblique parameter constraints and $m_{\mathrm{H}}, m_{\mathrm{A}}, m_{\mathrm{H}^{ \pm}}>150 \mathrm{GeV}$, and (iii) a number of constraints, to be discussed in the following, which involve both the quark Yukawa couplings and the scalar sector.

- Production $\times$ decay signal strengths of the $125 \mathrm{GeV} \mathrm{Hig}$ gs-like scalarh.

Agreement with the combined results of ATLAS and CMS from the LHC-Run I [39], together with additional data, involving in particular $\mathrm{h} \rightarrow b \bar{b}[42,43]$ from LHCRun II, constrains the scalar mixings $\mathcal{R}_{j 1}$ and the diagonal entries of the $N_{d}$ and $N_{u}$ matrices (see, for example, [44]). Notice that the requirement $\left|\mathcal{R}_{11}\right| \geq 0.9$ used in Sect. 4 to mimic coarsely the effect of these results is, of course, not imposed here.

- Neutral meson mixings.

One of the most relevant characteristics of the model is the presence of tree level flavour changing couplings of the neutral scalars: they produce the contributions to neutral meson mixing represented in Fig. 5a. They affect mass differences and $\mathrm{CP}$ violating observables $[23,45]$. For $B_{d}^{0}-\bar{B}_{d}^{0}$ and $B_{s}^{0}-\bar{B}_{s}^{0}$ we impose agreement with the mass differences $\Delta M_{B_{d}}, \Delta M_{B_{s}}$ and the mixing $\times$ decay $\mathrm{CP}$ asymmetries in $B_{d} \rightarrow J / \Psi K_{S}$ and $B_{S} \rightarrow J / \Psi \Phi$, respectively. For $K^{0}-\bar{K}^{0}$, we impose that the scalar mediated short distance contribution to $M_{12}^{K}$ does not yield sizable contributions to $\epsilon_{K}$ and $\Delta M_{K}$; in particular, for $\Delta M_{K}$, we require $2\left|M_{12}^{K}\right|_{S F C N C}<\Delta M_{K}$. For $D^{0}-\bar{D}^{0}$, we impose, similarly, that the short distance contribution to $M_{12}^{D}$ verifies $\left|M_{12}^{D}\right|<3 \times 10^{-2} \mathrm{ps}^{-1}$. In summary, neutral meson mixings constrain scalar mixings $\mathcal{R}_{i j}$ and masses, together with off-diagonal entries of $N_{d}$ and the 12, 21, elements of $N_{u}$.

Besides the SM one loop contribution, we only consider the scalar mediated tree level contributions to the Wilson coefficients of the different operators of interest; their QCD evolution from the electroweak scale to low energies follows [46-48]. For the operator matrix elements and bag factors, we use [49,50] (see also [51]).

$-b \rightarrow s \gamma$.

One loop diagrams like, for example, the ones in Fig. 5b,
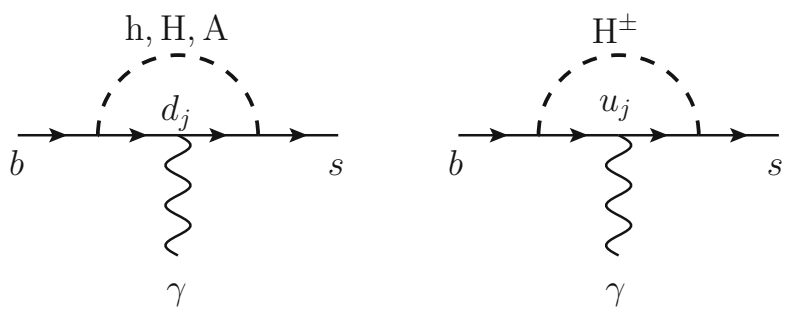

(b)

contribute to $\operatorname{Br}\left(B \rightarrow X_{s} \gamma\right)$, and further constrain $N_{u}$ and $N_{d}$, the neutral scalar mixings and masses, and $m_{\mathrm{H}^{ \pm}}$ (in the previous constraints $m_{\mathrm{H}^{ \pm}}$does only appear in the one loop $\mathrm{h} \rightarrow \gamma \gamma$ amplitude). Details of the calculation follow [52,53].

- Rare top decays $t \rightarrow \mathrm{h} q$.

Current bounds [54-56] on $t \rightarrow \mathrm{h} c, \mathrm{~h} u$ are at the $10^{-3}$ level, and have been included in the analysis.

- In addition to the previous constraints, we also consider the impact of imposing a sufficient suppression of $\theta_{\mathrm{QFD}}^{(1-\text { loop })}$ in order to avoid the strong CP problem (see Sect. 3.5). In the results presented in the following section, the larger blue regions correspond to the allowed regions arising from all the constraints except $\theta_{\mathrm{QFD}}^{(1-\text { loop })}$ (that is, ignoring the strong $\mathrm{CP}$ problem); the smaller red regions correspond to the additional requirement that $\theta_{\mathrm{QFD}}^{(1-l o o p)}$ is sufficiently suppressed. The later illustrate that, even if the focus of this model is not on the strong $\mathrm{CP}$ problem, if one insists on avoiding it, the model can do so in different regions of parameter space (as already mentioned, through fine-tuning, of course).

The analysis has two main goals:

1. to establish that the model is viable after a reasonable set of constraints is imposed;

2. to explore the prospects for the observation of some definite non-SM signal. We concentrate in particular on flavour changing decays $t \rightarrow \mathrm{h} c, \mathrm{~h} u$ and $\mathrm{h} \rightarrow b s, b d$, of interest, respectively, for the LHC and the ILC [57]. These are the most interesting tree level induced neutral flavour changing decays, since $\mathrm{h} \rightarrow u c, d s$ are more suppressed by the light fermion mass factors in $N_{u}$ and $N_{d}$ (in addition, the experimental analysis is also more difficult having only light quarks in the final state).

We also consider a representative low energy observable, the time dependent $\mathrm{CP}$ violating asymmetry in $B_{S} \rightarrow J / \Psi \Phi, A_{J / \Psi \Phi}^{C P}$, for which the SM prediction is $A_{J / \Psi \Phi}^{C P} \simeq-0.04$, while recent results, for example in [58], give $-0.030 \pm 0.033$, leaving significant room for New Physics contributions (on that respect, for the 


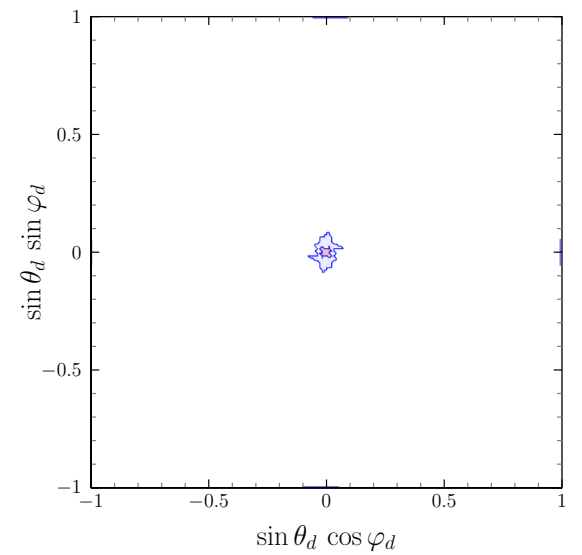

(a) $s_{\theta_{d}} s_{\varphi_{d}}$ vs. $s_{\theta_{d}} c_{\varphi_{d}}$

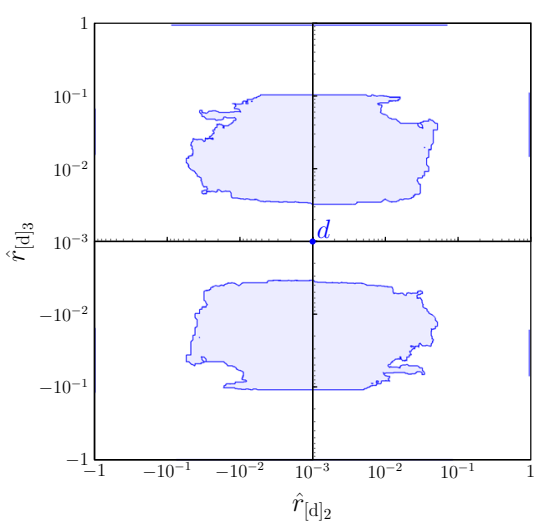

(d) $\hat{r}_{[\mathrm{d}] 3}$ vs. $\hat{r}_{[\mathrm{d}] 2}$

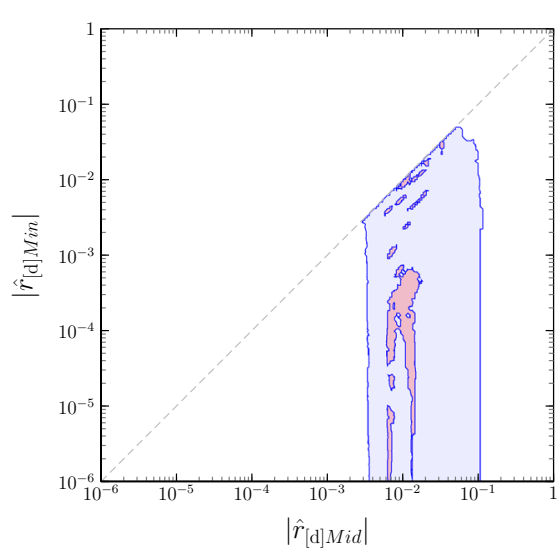

(b) $\left|\hat{r}_{[\mathrm{d}] M i n}\right|$ vs. $\left|\hat{r}_{[\mathrm{d}] M i d}\right|$.

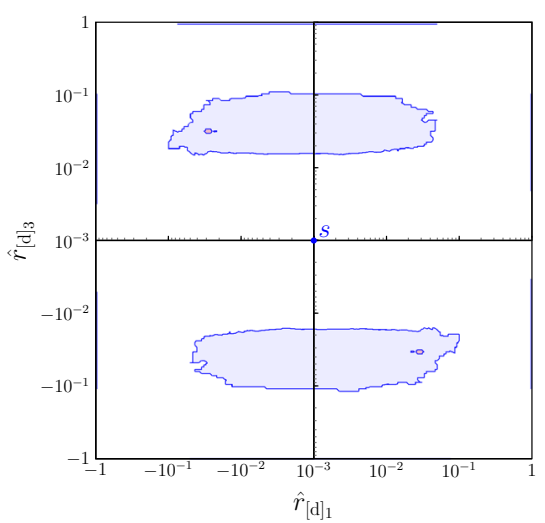

(e) $\hat{r}_{[\mathrm{d}] 3}$ vs. $\hat{r}_{[\mathrm{d}] 1}$

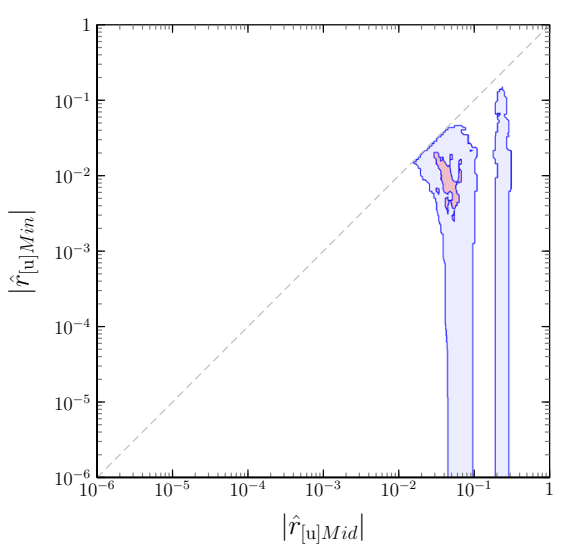

(c) $\left|\hat{r}_{[\mathrm{u}] M i n}\right|$ vs. $\left|\hat{r}_{[\mathrm{u}] M i d}\right|$.

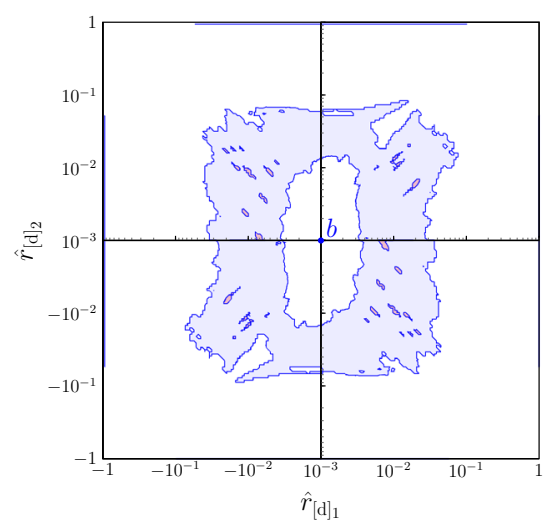

(f) $\hat{r}_{[\mathrm{d}] 2}$ vs. $\hat{r}_{[\mathrm{d}] 1}$

Fig. 6 Regions allowed at $99 \% \mathrm{CL}$ by the requirements of the full analysis (in blue, without requirements on $\theta_{\mathrm{QFD}}^{(1-l o o p)}$; in red, requiring $\theta_{\mathrm{QFD}}^{(1-l o o p)}<$ $10^{-10}$ )

moment it is the size of the uncertainty which is more relevant, rather than the central values of different recent measurements).

Further implications for the phenomenology of $\mathrm{H}, \mathrm{A}$ and $\mathrm{H}^{ \pm}$, in particular for the observation of these new scalars at the LHC, vary significantly between allowed regions in the parameter space of the model. The pattern of relevant decay modes for each scalar depends drastically on (i) the details of the scalar sector itself, and (ii) the couplings to fermions, i.e. the values of the $N_{q}$ matrices. Depending on the values of the scalar masses, their ordering, and the mixing matrix $\mathcal{R}$, the most relevant decays into gauge bosons and/or other scalars change. Concerning fermions, the widths of the different decays into quarks depend, for the neutral scalars, on both $\mathcal{R}$ and the $N_{q}$ matrices following Eq. (79), while for the charged scalar $\mathrm{H}^{ \pm}$they depend on $N_{q}$ alone, following Eq. (80). In addition, the couplings of the scalars with leptons, which we have not discussed in this work, ${ }^{7}$ would be neces-

\footnotetext{
7 The fact that we have not included a description of the leptonic sector prevents (i) the use of constraints such as, for example, $\operatorname{Br}\left(B_{S} \rightarrow\right.$
}

sary in order to include the decays into leptons, which also have to be considered: besides the direct interest as final states in different searches, they are required in order to know the complete pattern of decay branching ratios. As a result, direct "out of the box" application of constraints, like for example the ones provided by the HiggsBounds package [60], do not guarantee a consistent and complete coverage of the explored parameter space, and are not imposed here. ${ }^{8}$

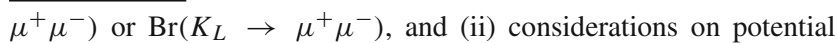
New Physics signals which involve leptons, as the so-called "B anomalies" [59].

8 As an illustration, consider for example three aspects that are assumed in [60]: (a) the only fermionic decays of $\mathrm{H}^{ \pm}$which are considered are into $c s, c b$ and $\tau v$ modes: besides the fact that the leptonic sector is not addressed here, these are not the dominant decays into quarks in large regions of parameters space (where, e.g., $t b$ decays are kinematically allowed and not parametrically suppressed); (b) no flavour changing decays of the neutral scalars are considered: in this model, they are necessarily present; (c) the narrow width approximation in production $x$ decay: once again, it does not hold in large regions of parameter space. 
Fig. 7 Regions allowed at 99\% CL by the requirements of the full analysis (see Fig. 6)

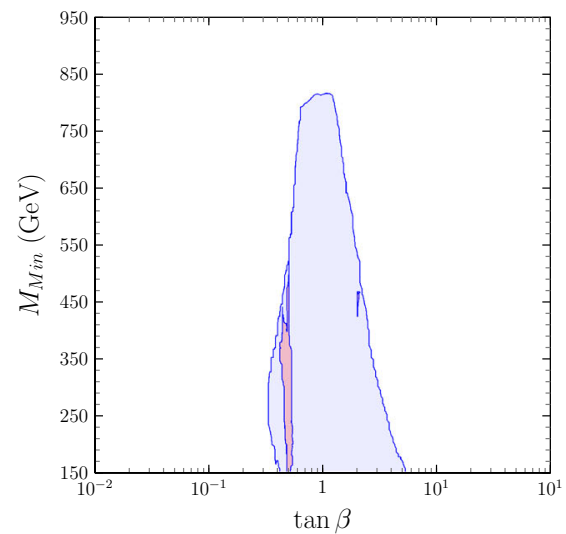

(a) $M_{M i n}$ vs. $\tan \beta$.

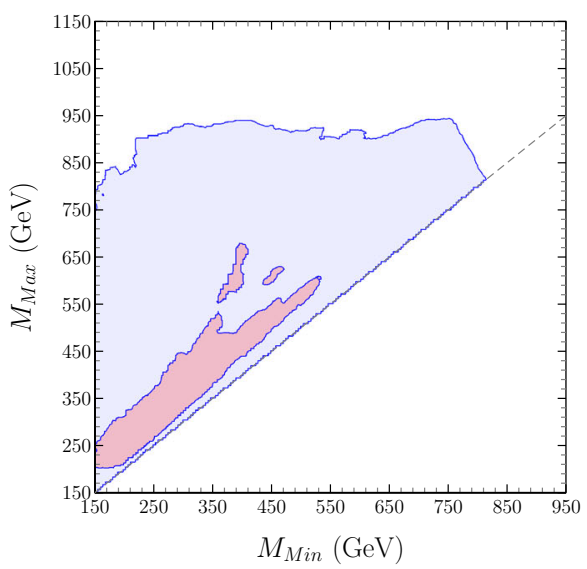

(c) $M_{\text {Max }}$ vs. $M_{M i n}$.

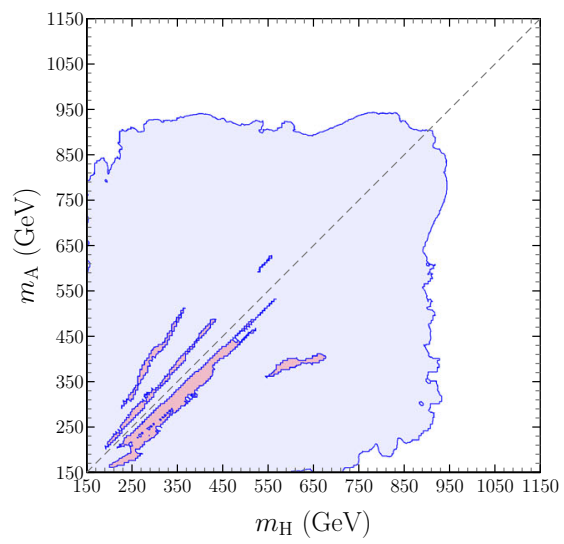

(b) $m_{\mathrm{A}}$ vs. $m_{\mathrm{H}}$.

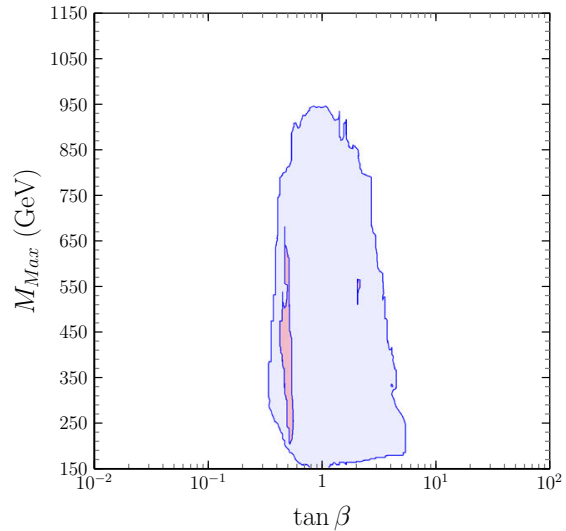

(b) $M_{\text {Max }}$ vs. $\tan \beta$.

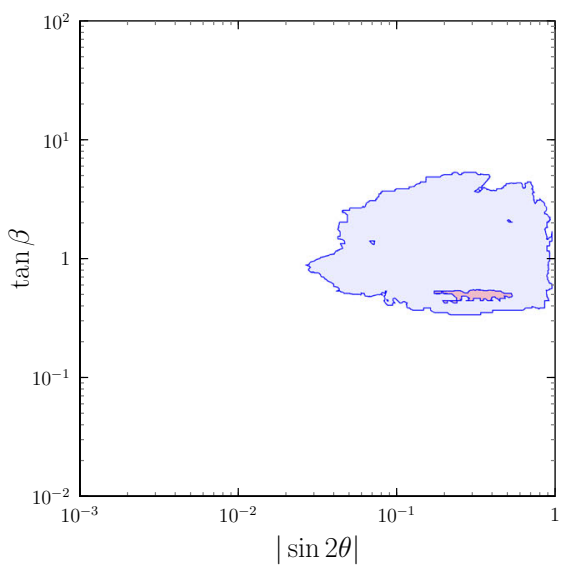

(d) $\tan \beta$ vs. $|\sin 2 \theta|$.

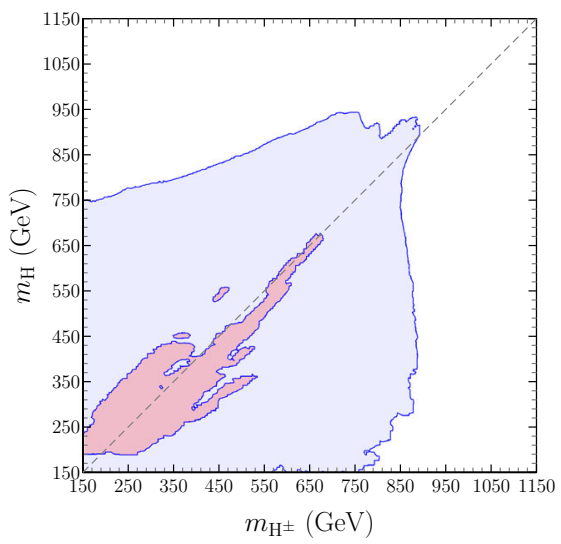

(c) $m_{\mathrm{H}}$ vs. $m_{\mathrm{H}^{ \pm}}$.

Fig. 8 Regions allowed at 99\% CL by the requirements of the full analysis (see Fig. 6)

\subsection{Results}

The main results of the full analysis are presented in Figs. 6, 7, 8, 9, 10 and 11.

Figure 6a corresponds to Fig. $2 \mathrm{~d}-\mathrm{f}$ of the analysis in Sect. 3: as one could anticipate, it is to be noticed that the allowed regions, where the model satisfies all the constraints, are much reduced with respect to the simple requirement of Sect. 3, i.e. just reproducing a realistic CKM matrix. In particular, the only allowed regions for $\theta_{d}$ and $\varphi_{d}$ correspond to having one component of $\hat{r}_{\text {[d] }}$ close to \pm 1 (that is close to the points $(0, \pm 1),( \pm 1,0),(0,0)$ in Fig. $6 a)$, and the 


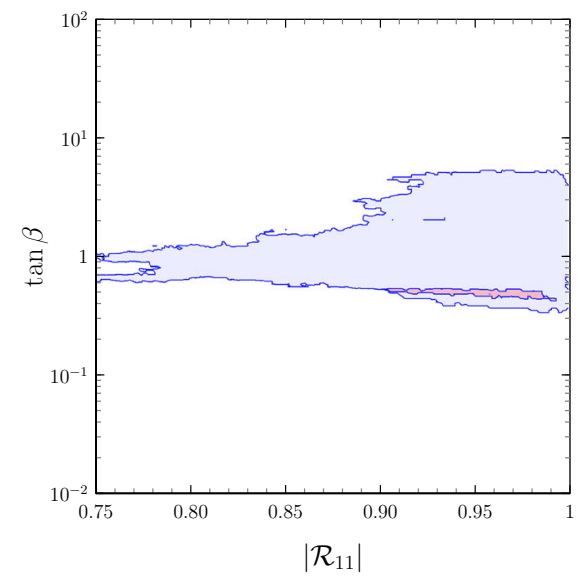

(a) $t_{\beta}$ vs. $\left|\mathcal{R}_{11}\right|$.

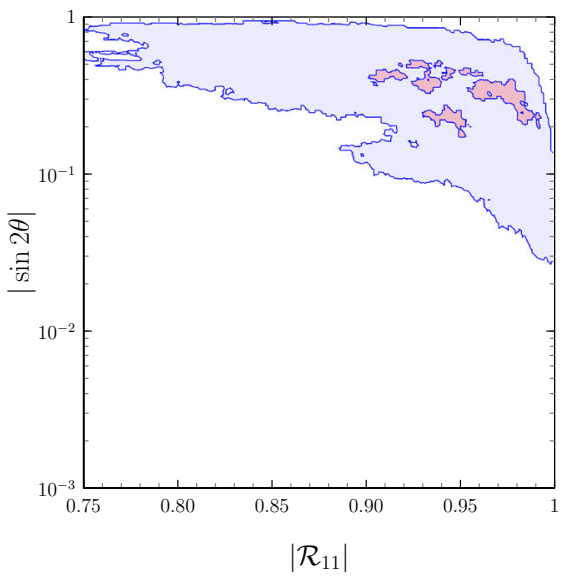

(b) $|\sin 2 \theta|$ vs. $\left|\mathcal{R}_{11}\right|$.

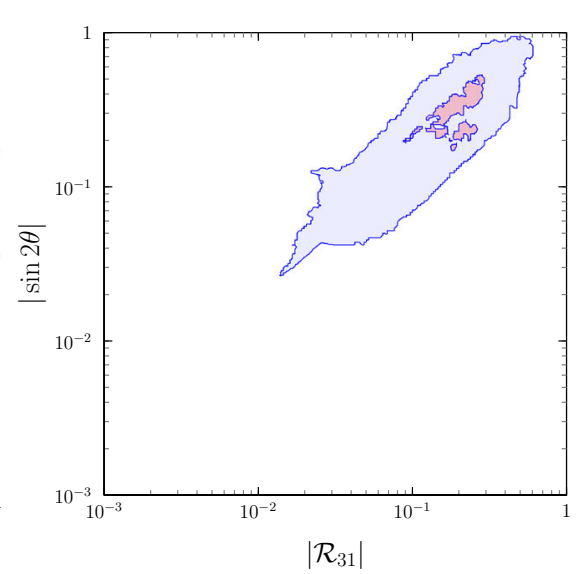

(c) $|\sin 2 \theta|$ vs. $\mathcal{R}_{31}$.

Fig. 9 Regions allowed at 99\% CL by the requirements of the full analysis (see Fig. 6)

Fig. 10 Region allowed at $99 \%$ CL by the requirements of the full analysis (see Fig. 6)

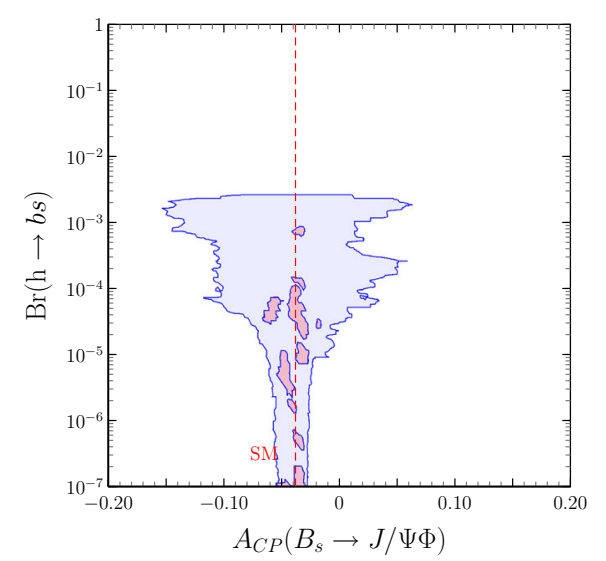

(a) $\operatorname{Br}(\mathrm{h} \rightarrow b s)$ vs. $A_{J / \Psi \Phi}^{C P}$.

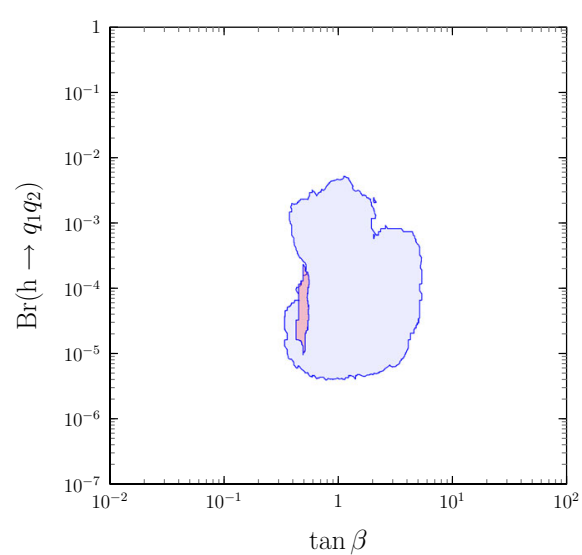

(b) $\operatorname{Br}\left(\mathrm{h} \rightarrow q_{1} q_{2}\right)$ vs. $t_{\beta}$. remaining two components much smaller: this naturally suppresses neutral flavour changing couplings, since they depend on the products of different components. As discussed in Sect. 3.2 and in Appendix A, without actually reaching that exact point, at which the CKM matrix becomes CP conserving. From this point of vue, those regions are "close to" (but not exactly) the different types of BGL models (as discussed in [12]), in which (i) tree SFCNC are absent in one of the quark sectors and (ii) the scalar potential does not permit spontaneous CP violation. This is clearly illustrated by Fig. 6d-f, that are enlargements of Fig. 6a with peculiar logarithmic scales where values below $10^{-3}$ have been collapsed to the central point. These central points correspond, respectively, to the flavour structures of the BGL models of types $d, s$ and $b$. For example, the $b$ BGL model corresponds to $\hat{r}_{[\mathrm{d}]}=(0,0, \pm 1)$. The figures show that the allowed regions exclude the BGL models, but remain close. Figure 6b, c correspond to Fig. 2b, c in Sect. 3.4: the range of allowed values for $\left|\hat{r}_{[\mathrm{d}] \text { Mid }}\right|,\left|\hat{r}_{[\mathrm{u}] \text { Mid }}\right|$ is reduced in the full analysis, in particular the largest allowed values are now smaller than 0.3.

In some cases it is difficult to distinguish in Fig. 6 the red regions, where $\theta_{\mathrm{QFD}}^{(1-l o o p)}<10^{-10}$ has been imposed: this is due to the particular scales used, in particular, in Fig. $6 \mathrm{~d}-\mathrm{f}$.

Figure 7 corresponds to Fig. 3 of the analysis of the scalar sector in Sect. 4. It is clear that the constraints of the full analysis reduce the available room for $t_{\beta}$, leaving only $1 / 4<$ $t_{\beta}<4$. Furthermore, the allowed region in $M_{\text {Max }}$ vs. $M_{\text {Min }}$ in Fig. $7 \mathrm{c}$ is slightly reduced with respect to Fig. 3c. Notice in particular that the region with all new scalars light, i.e. $M_{\text {Max }}<250 \mathrm{GeV}$, is now almost excluded. On the contrary, the largest values of $M_{M a x}$ and $M_{M i n}$ coincide with those in Sect. 4 , that is, they are still limited by the requirements on the scalar sector itself. The same comments apply to Fig. 8, which corresponds to Fig. 4 of the analysis of Sect. 4. Notice that $|\sin 2 \theta|$ is now required to be in the range $[0.03 ; 1.0]$. 


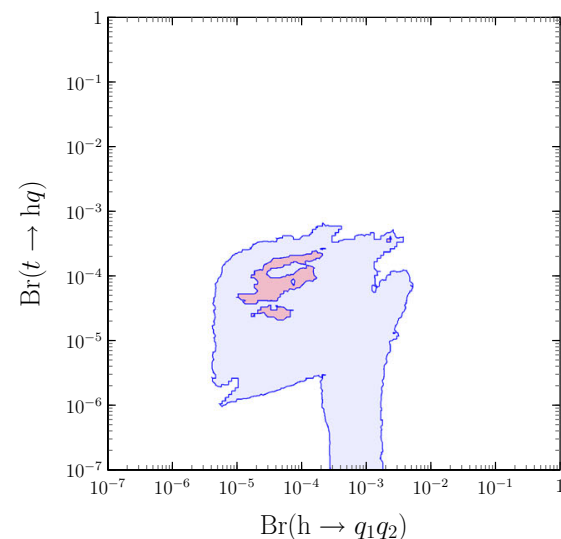

(a) $t \rightarrow \mathrm{h} q$ vs. $\mathrm{h} \rightarrow q_{1} q_{2}$.

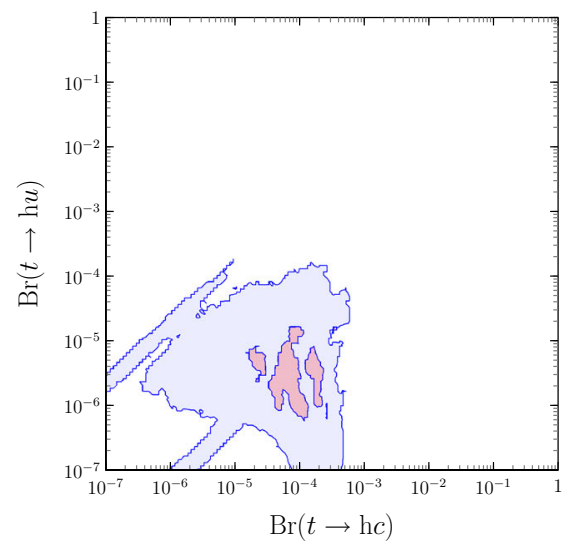

(d) $t \rightarrow \mathrm{h} u$ vs. $t \rightarrow \mathrm{h} c$.

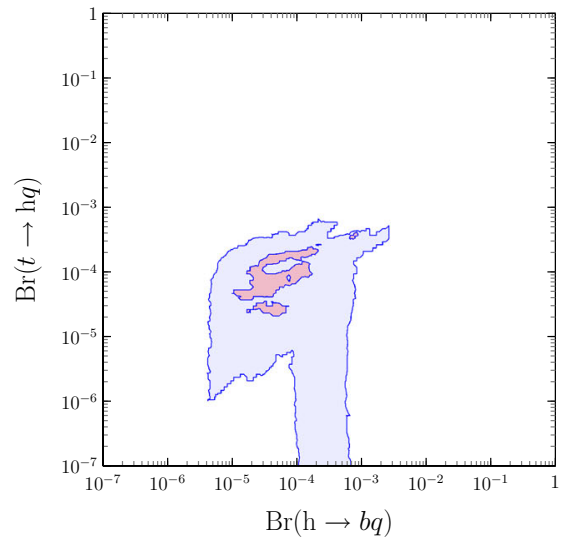

(b) $t \rightarrow \mathrm{h} q$ vs. $\mathrm{h} \rightarrow b q$.

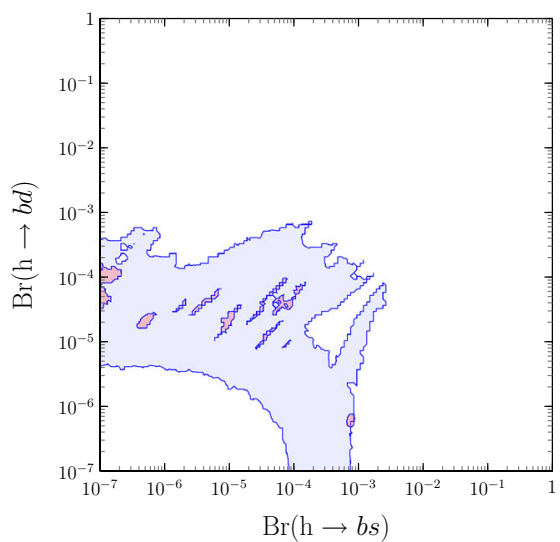

(e) $\mathrm{h} \rightarrow b d$ vs. $\mathrm{h} \rightarrow b s$.

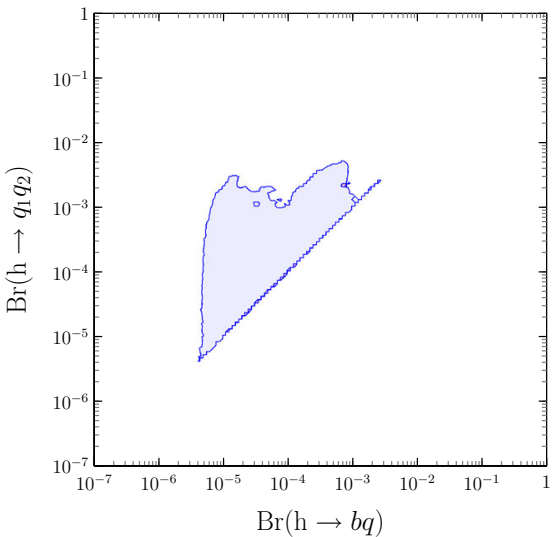

(c) $\mathrm{h} \rightarrow q_{1} q_{2}$ vs. $\mathrm{h} \rightarrow b q$.

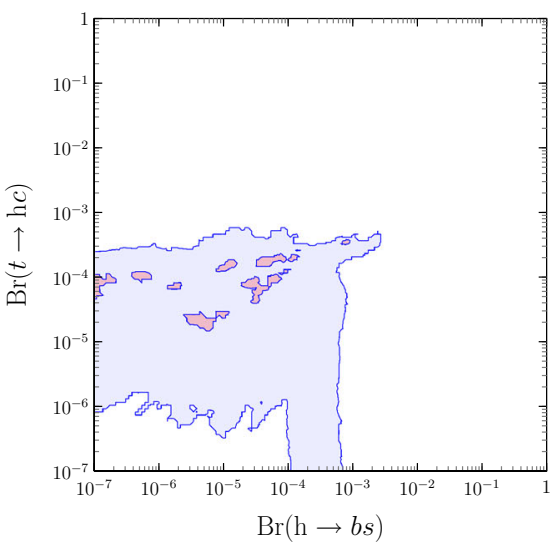

(f) $t \rightarrow \mathrm{h} c$ vs. $\mathrm{h} \rightarrow b s$.

Fig. 11 Regions allowed at $99 \%$ CL by the requirements of the full analysis (see Fig. 6)

Figure 9a shows that deviations from $\left|\mathcal{R}_{11}\right|=1$ can be achieved for almost all values of $t_{\beta}$ within the allowed range, while Fig. 9b, c illustrate, as expected, that for large values of $|\sin 2 \theta|, \mathcal{R}_{31}$ (which controls the amount of pseudoscalar $I^{0}$ entering $\mathrm{h}$ ), reaches the larger allowed values, while $\left|\mathcal{R}_{11}\right|$ is reduced. Notice in particular that, overall, $\left|\mathcal{R}_{31}\right| \geq 10^{-2}$ and that values as large as $\left|\mathcal{R}_{31}\right| \sim 0.4$ are allowed. In any case, even if $\left|\mathcal{R}_{11}\right|$ can reach values very close to $1,\left|\mathcal{R}_{11}\right|<1$.

Finally, Figs. 10 and 11 illustrate some New Physics prospects in different flavour changing neutral transitions.

Figure 10 a shows ${ }^{9} \operatorname{Br}(\mathrm{h} \rightarrow b s)$ vs. $A_{J / \Psi \Phi}^{C P}$; it is interesting to notice that: (i) $\mathrm{Br}(\mathrm{h} \rightarrow b s)$ can reach values as large as $10^{-2}$, relevant for searches at the ILC, and (ii) significant deviations of the $\mathrm{SM}$ expectation $A_{J / \Psi \Phi}^{C P} \simeq-0.036$ can arise. An interesting correlation among New Physics effects follows: $A_{J / \Psi \Phi}^{C P}$ values neatly different from SM expectations (the dashed vertical line in Fig. 10a) would necessarily require values of $\operatorname{Br}(\mathrm{h} \rightarrow b s)$ in the range $10^{-4}-10^{-2}$. The

\footnotetext{
9 The notation is $\operatorname{Br}(\mathrm{h} \rightarrow b s) \equiv \operatorname{Br}(\mathrm{h} \rightarrow \bar{b} s+b \bar{s}), \operatorname{Br}(\mathrm{h} \rightarrow b d) \equiv$ $\mathrm{Br}(\mathrm{h} \rightarrow \bar{b} d+b \bar{d})$, etc.
}

origin of such a correlation is clear: the tree level couplings that induce $\mathrm{h} \rightarrow b s$ at that level also contribute significantly to the dispersive amplitude $M_{12}^{B_{s}}$ in $B_{s}^{0}-\bar{B}_{s}^{0}$ mixing, changing its phase while maintaining $\left|M_{12}^{B_{s}}\right|$ (i.e. $\Delta M_{B_{s}}$ ). According to the discussion on the connection of SFCNC and CP violation in Sect. 3.2, tree level SFCNC should give

$$
\begin{aligned}
& \mathrm{Br}(t \rightarrow \mathrm{h} c)+\mathrm{Br}(t \rightarrow \mathrm{h} u)+\operatorname{Br}(\mathrm{h} \rightarrow c u) \neq 0 \quad \text { and } \\
& \operatorname{Br}(\mathrm{h} \rightarrow b s)+\operatorname{Br}(\mathrm{h} \rightarrow b d)+\operatorname{Br}(\mathrm{h} \rightarrow s d) \neq 0 .
\end{aligned}
$$

We introduce

$$
\begin{aligned}
& \mathrm{Br}(t \rightarrow \mathrm{h} q) \equiv \operatorname{Br}(t \rightarrow \mathrm{h} c)+\operatorname{Br}(t \rightarrow \mathrm{h} u), \\
& \operatorname{Br}(\mathrm{h} \rightarrow b q) \equiv \operatorname{Br}(\mathrm{h} \rightarrow b s)+\operatorname{Br}(\mathrm{h} \rightarrow b d), \\
& \operatorname{Br}\left(\mathrm{h} \rightarrow q_{1} q_{2}\right) \equiv \operatorname{Br}(\mathrm{h} \rightarrow b q)+\operatorname{Br}(\mathrm{h} \rightarrow s d)+\operatorname{Br}(\mathrm{h} \rightarrow c u) .
\end{aligned}
$$

Concentrating on the decays of h, Eq. (88) implies, for the total rate of flavour changing decays of $\mathrm{h} \operatorname{Br}\left(\mathrm{h} \rightarrow q_{1} q_{2}\right)$, $\operatorname{Br}\left(\mathrm{h} \rightarrow q_{1} q_{2}\right) \neq 0$. Figure $10 \mathrm{~b}$ clearly shows that in any case $5 \times 10^{-6} \leq \mathrm{Br}\left(\mathrm{h} \rightarrow q_{1} q_{2}\right) \leq 2 \times 10^{-2}$. 
Figure 11 shows different correlations among the branching ratios of flavour changing transitions involving $h$. It is important to notice that these New Physics signals are not confined to one particular sector (up or down type quarks) and that the largest allowed rates can be achieved for the transitions with second and third generation quarks, $t \rightarrow \mathrm{h} c$ and $\mathrm{h} \rightarrow b s$. Notice in particular that for $t \rightarrow \mathrm{h} c$, the LHC bounds at the level of $10^{-3}$ do play a role in limiting the allowed regions. Of course, the remaining transitions, $t \rightarrow \mathrm{h} u$ and $\mathrm{h} \rightarrow b d, s d, c u$ are also interesting: even if the largest values of their rates are smaller than the largest values allowed for $t \rightarrow \mathrm{h} c$ and $\mathrm{h} \rightarrow b s$, in some regions of the parameter space they have larger rates than $t \rightarrow \mathrm{h} c$ and $\mathrm{h} \rightarrow b s$, and they can also be within experimental reach.

\section{Conclusions}

In this paper we have addressed the question: is it possible to construct a realistic model with spontaneous $\mathrm{CP}$ violation, in the framework of a minimal two Higgs doublet extension of the Standard Model? We show that this is indeed possible. In order to accomplish this task, one has to surmount enormous obstacles, like having a natural suppression of SFCNC and generating a complex CKM matrix from the vacuum phase, with the correct strength of the invariant measure of the amount of $\mathrm{CP}$ violation in the quark mixing matrix.

We have shown that a minimal scenario is phenomenological viable, through the introduction of a flavoured $\mathbb{Z}_{2}$ symmetry, where one of the three quark families is odd under $\mathbb{Z}_{2}$ while the other two are even. A remarkable feature of the model is its prediction of New Physics which can be discovered at the LHC. More precisely, the model predicts that all the new scalars have a mass below $950 \mathrm{GeV}$ with at least one of the masses below $750 \mathrm{GeV}$. This prediction is obtained through a thorough study of the constraints arising from the $125 \mathrm{GeV}$ Higgs signals, the size of neutral meson mixings, the size of $b \rightarrow s \gamma$, and reproducing a correct CKM matrix, including the size of $\mathrm{CP}$ violation. Constraints from the electroweak oblique parameters, and perturbative unitarity and boundedness of the scalar potential are also included.

We encounter a deep connection between the generation of a complex CKM matrix from a vacuum phase and the necessary appearance of SFCNC. In the New Physics predictions, we give special emphasis to processes like $t \rightarrow \mathrm{h} c, \mathrm{~h} u$, $h \rightarrow b s, b d$, which are relevant for the LHC and the ILC. Interestingly, there is still room for important New Physics contributions to the phase of $B_{s}^{0}-\bar{B}_{s}^{0}$ mixing.

In the present model of SCPV, none of the new scalars can be heavier than $1 \mathrm{TeV}$, and the presence of SFCNC cannot be avoided. The experimental constraints select regions in parameter space where the SFCNC are kept under control, as happens in BGL models. It is indeed remarkable that these allowed regions are located close to BGL models: for example, in the neighbourhood of a down-type BGL flavour structure, we almost do not have SFCNC in the down sector while the SFCNC in the up sector are of the Minimal Flavour Violating type [6]. Apparently these are the only regions within this model, where one can have an effective suppression of the dangerous SFCNC.

Acknowledgements This work is partially supported by Spanish MINECO under Grant FPA2017-85140-C3-3-P and by the Severo Ochoa Excellence Center Project SEV-2014-0398, by Generalitat Valenciana under grant GVPROMETEOII 2014-049 and by Fundação para a Ciência e a Tecnologia (FCT, Portugal) through the projects CERN/FIS-PAR/0004/2017, CFTP-FCT Unit 777 (UID/FIS/00777/ 2013) and PTDC/FIS-PAR/29436/2017 which are partially funded through POCTI (FEDER), COMPETE, QREN and EU. MN acknowledges support from FCT through postdoctoral Grant SFRH/BPD/112999 12015 .

Data Availability Statement This manuscript has no associated data or the data will not be deposited. [Authors' comment: The manuscript has no associated data.]

Open Access This article is distributed under the terms of the Creative Commons Attribution 4.0 International License (http://creativecomm ons.org/licenses/by/4.0/), which permits unrestricted use, distribution, and reproduction in any medium, provided you give appropriate credit to the original author(s) and the source, provide a link to the Creative Commons license, and indicate if changes were made.

Funded by $\mathrm{SCOAP}^{3}$.

\section{A SFCNC and CP Violating CKM}

In Sect. 3.2 we have addressed the incompatibility between a $\mathrm{CP}$ violating CKM matrix and the absence of tree level SFCNC in one quark sector, in this model. In this appendix we provide a simple proof that completes the discussion.

Let us consider the case of the down quark sector. According to Eqs. (22) and (23),

$$
\begin{aligned}
N_{d}= & {\left[t_{\beta} \mathbf{1}-\left(t_{\beta}+t_{\beta}^{-1}\right) \mathcal{O}_{L}^{d T} \mathrm{P}_{3} \mathcal{O}_{L}^{d}\right] M_{d}, } \\
& {\left[\mathcal{O}_{L}^{d T} \mathrm{P}_{3} \mathcal{O}_{L}^{d}\right]_{i j}=\hat{r}_{[\mathrm{d}] i} \hat{r}_{[\mathrm{d}] j} . }
\end{aligned}
$$

Tree level SFCNC in the down sector are absent when $\mathcal{O}_{L}^{d T} \mathrm{P}_{3} \mathcal{O}_{L}^{d}$ is diagonal, that is $\hat{r}_{[\mathrm{d}] i}=\delta_{i k}$ for some $k$ (1 or 2 or 3$)$. In that case,

$\left[\mathcal{O}_{L}^{d T} \mathrm{P}_{3} \mathcal{O}_{L}^{d}\right]_{i j}=\delta_{i k} \delta_{j k}=\delta_{i k} \delta_{i j}=\left[\mathrm{P}_{k}\right]_{i j}$

with the projectors

$P_{1}=\left(\begin{array}{lll}1 & 0 & 0 \\ 0 & 0 & 0 \\ 0 & 0 & 0\end{array}\right), \quad P_{2}=\left(\begin{array}{lll}0 & 0 & 0 \\ 0 & 1 & 0 \\ 0 & 0 & 0\end{array}\right), \quad P_{3}=\left(\begin{array}{lll}0 & 0 & 0 \\ 0 & 0 & 0 \\ 0 & 0 & 1\end{array}\right)$.

On the other hand, the CKM matrix in Eq. (33) reads in that case 


$$
\begin{aligned}
V & =\mathcal{O}_{L}^{u T}\left[\mathbf{1}+\left(e^{i 2 \theta}-1\right) \mathrm{P}_{3}\right] \mathcal{O}_{L}^{d}=R\left[\mathbf{1}+\left(e^{i 2 \theta}-1\right) \mathcal{O}_{L}^{d T} \mathrm{P}_{3} \mathcal{O}_{L}^{d}\right] \\
& =R\left[\mathbf{1}+\left(e^{i 2 \theta}-1\right) \mathrm{P}_{k}\right],
\end{aligned}
$$

it is the product of a real orthogonal matrix $R$ and a diagonal matrix of phases, and hence $\mathrm{CP}$ conserving.

For the up quark sector, the reasoning is analogous: the absence of tree level SFCNC requires $\mathcal{O}_{L}^{u T} \mathrm{P}_{3} \mathcal{O}_{L}^{u}$ to be diagonal in

$$
\begin{aligned}
& N_{u}=\left[t_{\beta} \mathbf{1}-\left(t_{\beta}+t_{\beta}^{-1}\right) \mathcal{O}_{L}^{u T} \mathrm{P}_{3} \mathcal{O}_{L}^{u}\right] M_{u}, \\
& {\left[\mathcal{O}_{L}^{u T} \mathrm{P}_{3} \mathcal{O}_{L}^{u}\right]_{i j}=\hat{r}_{[\mathrm{u}] i} \hat{r}_{[\mathrm{u}] j},}
\end{aligned}
$$

that is $\hat{r}_{[\mathrm{u}] i}=\delta_{i k}$ for some $k$, in which case $\left[\mathcal{O}_{L}^{u T} \mathrm{P}_{3} \mathcal{O}_{L}^{u}\right]_{i j}=$ $\left[\mathrm{P}_{k}\right]_{i j}$ and

$$
V=\left[\mathbf{1}+\left(e^{i 2 \theta}-1\right) \mathcal{O}_{L}^{u T} \mathrm{P}_{3} \mathcal{O}_{L}^{u}\right] R=\left[\mathbf{1}+\left(e^{i 2 \theta}-1\right) \mathrm{P}_{k}\right] R,
$$

with the CKM matrix the product of a diagonal matrix of phases and a real orthogonal matrix $R$, hence $\mathrm{CP}$ conserving again.

\section{B Scalar potential}

In this appendix we discuss different aspects concerning the scalar potential of Sect. 4: in B.1 the election of a convenient set of basic parameters, in B.2 boundedness (from below) of the potential, then perturbativity requirements in B.3, and finally, in B.4, a simple proof that $\lambda_{5}>0$ is a necessary condition in the present scenario.

\section{B.1 Independent parameters}

It is important to discuss the number and nature of the independent parameters of interest in the scalar sector. The goal is to adopt the most convenient choice for them. Through the minimization conditions of Sect. 4.1, it is already clear that one can trade the three quadratic coefficients $\mu_{i j}^{2}$ for $v^{2}, \beta$ and $\theta$, and set $v=246 \mathrm{GeV}$. At this stage one could already consider a set of values for $\left\{\lambda_{j}\right\}, j=1, \ldots, 5$, compute $m_{\mathrm{H}^{ \pm}}$, the mass matrix $\mathcal{M}_{0}^{2}$, and from $\mathcal{M}_{0}^{2}$, obtain, at least numerically, $m_{\mathrm{h}}^{2}, m_{\mathrm{H}}^{2}, m_{\mathrm{A}}^{2}$ and the mixings $\mathcal{R}$. Of course, one would then need to impose appropriate conditions: for example $m_{\mathrm{h}}^{2}>0$, $m_{\mathrm{H}}^{2}>0, m_{\mathrm{A}}^{2}>0$. This is hardly the most convenient strategy, since, besides the computational toll, one would like for example to impose $m_{\mathrm{h}}=125 \mathrm{GeV}$. The phenomenological conditions in Sect. 6.1 can be imposed afterwards. With this in mind, one would prefer to have (beside $\beta$ and $\theta$ ), $m_{\mathrm{h}}^{2}, m_{\mathrm{H}}^{2}$, $m_{\mathrm{A}}^{2}, m_{\mathrm{H}^{ \pm}}^{2}$ and three angles $\alpha_{j}$ describing $\mathcal{R}$ as parameters, and the different $\lambda_{j}$ expressed in terms of them.

We have already noticed that $\lambda_{4}$ can be traded for $m_{\mathrm{H}^{ \pm}}^{2}$ and $\lambda_{5}$ using Eq. (68); this leaves four quantities, $\lambda_{1}, \lambda_{2}$, $\lambda_{345}$ and $\lambda_{5}$, that, together with $\beta$ and $\theta$, determine $\mathcal{M}_{0}^{2}$ (i.e. six different matrix elements). On the other hand, in $\mathcal{M}_{0}^{2}$, we have three masses, $m_{\mathrm{h}}^{2}, m_{\mathrm{H}}^{2}, m_{\mathrm{A}}^{2}$, while $\mathcal{R}$ requires three parameters: six quantities. It is to be expected that they cannot be chosen independently. One simple procedure that can be adopted is the following:

1. equating elements $\left[\mathcal{M}_{0}^{2}\right]_{13},\left[\mathcal{M}_{0}^{2}\right]_{23}$ and $\left[\mathcal{M}_{0}^{2}\right]_{33}$ in Eq. (70) with their expressions in terms of $m_{\mathrm{h}}^{2}, m_{\mathrm{H}}^{2}, m_{\mathrm{A}}^{2}$, and $\mathcal{R}$, they can be read as a linear system in $\lambda_{5}, m_{\mathrm{H}}^{2}$, $m_{\mathrm{A}}^{2}$ which can be solved, giving them in terms of $m_{\mathrm{h}}^{2}$, $\mathcal{R}$ and of course $\beta, v^{2}$ and $\theta$;

2. then, equating elements $\left[\mathcal{M}_{0}^{2}\right]_{11},\left[\mathcal{M}_{0}^{2}\right]_{22}$ and $\left[\mathcal{M}_{0}^{2}\right]_{12}$ in Eq. (70) with their expressions in terms of $m_{\mathrm{h}}^{2}, m_{\mathrm{H}}^{2}$, $m_{\mathrm{A}}^{2}$, and $\mathcal{R}$, they can be read as a linear system in $\lambda_{1}$, $\lambda_{2}$ and $\lambda_{345}$, which can also be solved, giving them in terms of $m_{\mathrm{h}}^{2}, \mathcal{R}, \beta, v^{2}$ and $\theta$;

3. $\lambda_{4}$ is simply given by $\lambda_{4}=\lambda_{5}-m_{\mathrm{H}^{ \pm}}^{2} / v^{2}$ with $\lambda_{5}$ already known;

4. with $\lambda_{345}, \lambda_{4}$ and $\lambda_{5}$ known, $\lambda_{3}$ is trivially $\lambda_{3}=\lambda_{345}+$ $\lambda_{5}-\lambda_{4}$.

Summarising: with these simple steps, for given values of $\beta$, $v^{2}, \theta, m_{\mathrm{h}}^{2}, m_{\mathrm{H}^{ \pm}}^{2}$ and $\mathcal{R}$ (three $\alpha_{j}$ ), one can compute $m_{\mathrm{H}}^{2}, m_{\mathrm{A}}^{2}$ and all $\lambda_{j}, j=1$ to 5 . For that set of values to be acceptable, one should then require

- positive values of all masses $\left(m_{\mathrm{h}}^{2}\right.$ and $m_{\mathrm{H}^{ \pm}}^{2}$ can be chosen and thus only $m_{\mathrm{H}}^{2}>0$ and $m_{\mathrm{A}}^{2}>0$ have to be checked),

- boundedness from below of $\mathscr{V}\left(\Phi_{1}, \Phi_{2}\right)$ and absolute minimum for $\left\{v^{2}, \beta, \theta\right\}$,

- perturbative unitarity requirements on $\lambda$ 's.

In order to illustrate the procedure to express $m_{\mathrm{A}}^{2}, m_{\mathrm{H}}^{2}$ and all $\lambda_{j}$ in terms of the basic set of parameters $\left\{v^{2}, \beta, \theta, m_{\mathrm{h}}^{2}, m_{\mathrm{H}^{ \pm}}^{2}\right.$, $\left.\alpha_{j}\right\}$, we start with the use of $\left[\mathcal{M}_{0}^{2}\right]_{13},\left[\mathcal{M}_{0}^{2}\right]_{23}$ and $\left[\mathcal{M}_{0}^{2}\right]_{33}$ to obtain $m_{\mathrm{A}}^{2}, m_{\mathrm{H}}^{2}$ and $\lambda_{5}$. One needs to equate those elements in Eq. (70) to

$$
\begin{aligned}
& {\left[\mathcal{M}_{0}^{2}\right]_{13}=m_{\mathrm{h}}^{2} \mathcal{R}_{11} \mathcal{R}_{31}+m_{\mathrm{H}}^{2} \mathcal{R}_{12} \mathcal{R}_{32}+m_{\mathrm{A}}^{2} \mathcal{R}_{13} \mathcal{R}_{33},} \\
& {\left[\mathcal{M}_{0}^{2}\right]_{23}=m_{\mathrm{h}}^{2} \mathcal{R}_{21} \mathcal{R}_{31}+m_{\mathrm{H}}^{2} \mathcal{R}_{22} \mathcal{R}_{32}+m_{\mathrm{A}}^{2} \mathcal{R}_{23} \mathcal{R}_{33},} \\
& {\left[\mathcal{M}_{0}^{2}\right]_{33}=m_{\mathrm{h}}^{2} \mathcal{R}_{31}^{2}+m_{\mathrm{H}}^{2} \mathcal{R}_{32}^{2}+m_{\mathrm{A}}^{2} \mathcal{R}_{33}^{2} .}
\end{aligned}
$$

From the orthonormality relations $\left(\mathcal{R}^{T} \mathcal{R}\right)_{i j}=\delta_{i j}$ we have

$$
\begin{aligned}
m_{\mathrm{h}}^{2} \mathcal{R}_{31} & =\mathcal{R}_{11}\left[\mathcal{M}_{0}^{2}\right]_{13}+\mathcal{R}_{21}\left[\mathcal{M}_{0}^{2}\right]_{23}+\mathcal{R}_{31}\left[\mathcal{M}_{0}^{2}\right]_{33}, \\
m_{\mathrm{H}}^{2} \mathcal{R}_{32} & =\mathcal{R}_{12}\left[\mathcal{M}_{0}^{2}\right]_{13}+\mathcal{R}_{22}\left[\mathcal{M}_{0}^{2}\right]_{23}+\mathcal{R}_{32}\left[\mathcal{M}_{0}^{2}\right]_{33}
\end{aligned}
$$

$$
m_{\mathrm{A}}^{2} \mathcal{R}_{33}=\mathcal{R}_{13}\left[\mathcal{M}_{0}^{2}\right]_{13}+\mathcal{R}_{23}\left[\mathcal{M}_{0}^{2}\right]_{23}+\mathcal{R}_{33}\left[\mathcal{M}_{0}^{2}\right]_{33},
$$


and thus

$$
\begin{aligned}
& m_{\mathrm{h}}^{2} \mathcal{R}_{31}=v^{2} \lambda_{5}\left[-s_{2 \beta} s_{2 \theta} \mathcal{R}_{11}-c_{2 \beta} s_{2 \theta} \mathcal{R}_{21}+2 s_{\theta}^{2} \mathcal{R}_{31}\right], \\
& m_{\mathrm{H}}^{2}=m_{\mathrm{h}}^{2} \frac{\mathcal{R}_{31}}{\mathcal{R}_{32}} \frac{\mathcal{R}_{12}\left[\mathcal{M}_{0}^{2}\right]_{13}+\mathcal{R}_{22}\left[\mathcal{M}_{0}^{2}\right]_{23}+\mathcal{R}_{32}\left[\mathcal{M}_{0}^{2}\right]_{33}}{\mathcal{R}_{11}\left[\mathcal{M}_{0}^{2}\right]_{13}+\mathcal{R}_{21}\left[\mathcal{M}_{0}^{2}\right]_{23}+\mathcal{R}_{31}\left[\mathcal{M}_{0}^{2}\right]_{33}}, \\
& m_{\mathrm{A}}^{2}=m_{\mathrm{h}}^{2} \frac{\mathcal{R}_{31}}{\mathcal{R}_{33}} \frac{\mathcal{R}_{13}\left[\mathcal{M}_{0}^{2}\right]_{13}+\mathcal{R}_{23}\left[\mathcal{M}_{0}^{2}\right]_{23}+\mathcal{R}_{33}\left[\mathcal{M}_{0}^{2}\right]_{33}}{\mathcal{R}_{11}\left[\mathcal{M}_{0}^{2}\right]_{13}+\mathcal{R}_{21}\left[\mathcal{M}_{0}^{2}\right]_{23}+\mathcal{R}_{31}\left[\mathcal{M}_{0}^{2}\right]_{33}} .
\end{aligned}
$$

The solution reads

$$
\begin{aligned}
& \lambda_{5}=\frac{m_{\mathrm{h}}^{2}}{2 v^{2}} \frac{\mathcal{R}_{31}}{s_{\theta}} \frac{1}{s_{\theta} \mathcal{R}_{31}-c_{\theta} c_{2 \beta} \mathcal{R}_{21}-c_{\theta} s_{2 \beta} \mathcal{R}_{11}}, \\
& m_{\mathrm{H}}^{2}=m_{\mathrm{h}}^{2} \frac{\mathcal{R}_{31}}{\mathcal{R}_{32}}\left[\frac{-c_{\theta} s_{2 \beta} \mathcal{R}_{12}-c_{\theta} c_{2 \beta} \mathcal{R}_{22}+s_{\theta} \mathcal{R}_{32}}{-c_{\theta} s_{2 \beta} \mathcal{R}_{11}-c_{\theta} c_{2 \beta} \mathcal{R}_{21}+s_{\theta} \mathcal{R}_{31}}\right], \\
& m_{\mathrm{A}}^{2}=m_{\mathrm{h}}^{2} \frac{\mathcal{R}_{31}}{\mathcal{R}_{33}}\left[\frac{-c_{\theta} s_{2 \beta} \mathcal{R}_{13}-c_{\theta} c_{2 \beta} \mathcal{R}_{23}+s_{\theta} \mathcal{R}_{33}}{-c_{\theta} s_{2 \beta} \mathcal{R}_{11}-c_{\theta} c_{2 \beta} \mathcal{R}_{21}+s_{\theta} \mathcal{R}_{31}}\right] .
\end{aligned}
$$

Next, equating elements $\left[\mathcal{M}_{0}^{2}\right]_{11},\left[\mathcal{M}_{0}^{2}\right]_{22}$ and $\left[\mathcal{M}_{0}^{2}\right]_{12}$ in Eq. (70) to

$$
\begin{aligned}
& {\left[\mathcal{M}_{0}^{2}\right]_{11}=m_{\mathrm{h}}^{2} \mathcal{R}_{11}^{2}+m_{\mathrm{H}}^{2} \mathcal{R}_{12}^{2}+m_{\mathrm{A}}^{2} \mathcal{R}_{13}^{2},} \\
& {\left[\mathcal{M}_{0}^{2}\right]_{22}=m_{\mathrm{h}}^{2} \mathcal{R}_{21}^{2}+m_{\mathrm{H}}^{2} \mathcal{R}_{22}^{2}+m_{\mathrm{A}}^{2} \mathcal{R}_{23}^{2},} \\
& {\left[\mathcal{M}_{0}^{2}\right]_{12}=m_{\mathrm{h}}^{2} \mathcal{R}_{11} \mathcal{R}_{21}+m_{\mathrm{H}}^{2} \mathcal{R}_{12} \mathcal{R}_{22}+m_{\mathrm{A}}^{2} \mathcal{R}_{13} \mathcal{R}_{23},}
\end{aligned}
$$

one can solve for $\lambda_{1}, \lambda_{2}$ and $\lambda_{345}$ :

$$
\begin{aligned}
\lambda_{1} & =\frac{1}{2 v^{2}}\left[\left[\mathcal{M}_{0}^{2}\right]_{11}+t_{\beta}^{2}\left[\mathcal{M}_{0}^{2}\right]_{22}+2 t_{\beta}\left[\mathcal{M}_{0}^{2}\right]_{12}\right]-\lambda_{5} c_{\theta}^{2} t_{\beta}^{2}, \\
\lambda_{2} & =\frac{1}{2 v^{2}}\left[\left[\mathcal{M}_{0}^{2}\right]_{11}+t_{\beta}^{-2}\left[\mathcal{M}_{0}^{2}\right]_{22}-2 t_{\beta}^{-1}\left[\mathcal{M}_{0}^{2}\right]_{12}\right]-\lambda_{5} c_{\theta}^{2} t_{\beta}^{-2}, \\
\lambda_{345} & =\frac{1}{2 v^{2}}\left[\left[\mathcal{M}_{0}^{2}\right]_{11}-\left[\mathcal{M}_{0}^{2}\right]_{22}+\left(t_{\beta}^{-1}-t_{\beta}\right)\left[\mathcal{M}_{0}^{2}\right]_{12}\right]-\lambda_{5} c_{\theta}^{2},
\end{aligned}
$$

that is

$$
\begin{aligned}
\lambda_{1}= & \frac{m_{\mathrm{h}}^{2}}{2 v^{2}}\left(\mathcal{R}_{11}-t_{\beta} \mathcal{R}_{21}\right)^{2}+\frac{m_{\mathrm{H}}^{2}}{2 v^{2}}\left(\mathcal{R}_{12}-t_{\beta} \mathcal{R}_{22}\right)^{2} \\
& +\frac{m_{\mathrm{A}}^{2}}{2 v^{2}}\left(\mathcal{R}_{13}-t_{\beta} \mathcal{R}_{23}\right)^{2}-\lambda_{5} c_{\theta}^{2} t_{\beta}^{2}, \\
\lambda_{2}= & \frac{m_{\mathrm{h}}^{2}}{2 v^{2}}\left(\mathcal{R}_{11}+t_{\beta}^{-1} \mathcal{R}_{21}\right)^{2}+\frac{m_{\mathrm{H}}^{2}}{2 v^{2}}\left(\mathcal{R}_{12}+t_{\beta}^{-1} \mathcal{R}_{22}\right)^{2} \\
& +\frac{m_{\mathrm{A}}^{2}}{2 v^{2}}\left(\mathcal{R}_{13}+t_{\beta}^{-1} \mathcal{R}_{23}\right)^{2}-\lambda_{5} c_{\theta}^{2} t_{\beta}^{-2}, \\
\lambda_{345}= & \frac{m_{\mathrm{h}}^{2}}{2 v^{2}}\left(\mathcal{R}_{11}^{2}-\mathcal{R}_{21}^{2}+\left(t_{\beta}^{-1}-t_{\beta}\right) \mathcal{R}_{11} \mathcal{R}_{21}\right) \\
& +\frac{m_{\mathrm{H}}^{2}}{2 v^{2}}\left(\mathcal{R}_{12}^{2}-\mathcal{R}_{22}^{2}+\left(t_{\beta}^{-1}-t_{\beta}\right) \mathcal{R}_{12} \mathcal{R}_{22}\right) \\
& +\frac{m_{\mathrm{A}}^{2}}{2 v^{2}}\left(\mathcal{R}_{13}^{2}-\mathcal{R}_{23}^{2}+\left(t_{\beta}^{-1}-t_{\beta}\right) \mathcal{R}_{13} \mathcal{R}_{23}\right)-\lambda_{5} c_{\theta}^{2},
\end{aligned}
$$

with $m_{\mathrm{H}}^{2}, m_{\mathrm{A}}^{2}$ and $\lambda_{5}$ in Eqs. (105)-(107). To complete the procedure, we just need to recall

$\lambda_{4}=\lambda_{5}-m_{\mathrm{H}^{ \pm}}^{2} / v^{2}, \quad \lambda_{3}=\lambda_{345}-\lambda_{4}+\lambda_{5}$.

\section{B.2 Boundedness and absolute minimum}

The conditions to be imposed on the resulting $\lambda_{j}$ 's for a scalar potential bounded from below are

$\lambda_{1}>0, \quad \lambda_{2}>0, \quad \sqrt{\lambda_{1} \lambda_{2}}>-\lambda_{3}, \quad \lambda_{345}>-\sqrt{\lambda_{1} \lambda_{2}}$.

Notice that with the expression of $\operatorname{det} \mathcal{M}_{0}^{2}$ in Eq. (74), with $\lambda_{5}>0$ (see Sect. B.4 below) it follows from $\operatorname{det} \mathcal{M}_{0}^{2}>0$ that

$\sqrt{\lambda_{1} \lambda_{2}}>\lambda_{345}>-\sqrt{\lambda_{1} \lambda_{2}}$.

One last concern on the scalar potential is the possibility that the local minimum for $\left\{v^{2}, \beta, \theta\right\}$ is not the absolute minimum of the potential, but instead a metastable minimum which can decay to the "true" absolute minimum (such a situation is sometimes dubbed the panic vacuum [61]). From general studies of the minimization problem in 2HDM [6164], it follows that $\left\{v^{2}, \beta, \theta\right\}$ and $\left\{v^{2}, \beta,-\theta\right\}$ (this discrete ambiguity arised already in Eq. (60)) give indeed the absolute minima of the potential.

\section{B.3 Perturbative unitarity}

Requiring perturbative unitarity of tree level scattering processes translates into the following bounds $[65,66]$ (one loop corrections in a CP conserving $2 \mathrm{HDM}$ scenario have been addressed in [67])

$\lambda_{1}+\lambda_{2} \pm \sqrt{\left(\lambda_{1}-\lambda_{2}\right)^{2}+4 \lambda_{5}^{2}}<\Lambda$,

$2\left(\lambda_{3}+\lambda_{4}\right)<\Lambda$,

$2\left(\lambda_{3}-\lambda_{4}\right)<\Lambda$,

$\lambda_{1}+\lambda_{2} \pm \sqrt{\left(\lambda_{1}-\lambda_{2}\right)^{2}+4 \lambda_{4}^{2}}<\Lambda$,

$2\left(\lambda_{3} \pm \lambda_{5}\right)<\Lambda$,

$3\left(\lambda_{1}+\lambda_{2}\right) \pm \sqrt{9\left(\lambda_{1}-\lambda_{2}\right)^{2}+4\left(2 \lambda_{3}+\lambda_{4}\right)^{2}}<\Lambda$,

$2\left(\lambda_{3}+2 \lambda_{4} \pm 3 \lambda_{5}\right)<\Lambda$,

with $\Lambda=16 \pi$.

B. $4 \lambda_{5}>0$

As anticipated in Sect. 4, the necessary condition $\lambda_{5}>0$ follows from a simple requirement on the scalar potential. If $V\left(v_{1}, v_{2}, \theta\right)$ is the absolute minimum of the potential, it is obviously necessary that $V\left(v_{1}, v_{2}, \theta\right)<V\left(v_{1}, v_{2}, 0\right)$ 
and $V\left(v_{1}, v_{2}, \theta\right)<V\left(v_{1}, v_{2}, \pi\right)$. Notice that, although $\left\{v_{1}, v_{2}, 0\right\}$ and $\left\{v_{1}, v_{2}, \pi\right\}$ fulfill Eq. (57), they do not fulfill Eqs. (58) and (59), that is, $V$ cannot have a minimum for $\left\{v_{1}, v_{2}, 0\right\},\left\{v_{1}, v_{2}, \pi\right\}$. Since the $\theta$-independent terms of $V$ are common to all three cases, we only need to analyse the $\theta$-dependent part, $V_{\theta}$.

$$
\begin{aligned}
V_{\theta}\left(v_{1}, v_{2},{ }_{\pi}^{0}\right) & =v_{1} v_{2}\left[ \pm \mu_{12}^{2}+\frac{1}{2} v_{1} v_{2} \lambda_{5}\right] \\
& =v_{1}^{2} v_{2}^{2} \lambda_{5}\left[\frac{1}{2} \mp 2 \cos \theta\right],
\end{aligned}
$$

while

$$
\begin{aligned}
V_{\theta}\left(v_{1}, v_{2}, \theta\right) & =v_{1} v_{2}\left[\mu_{12}^{2} \cos \theta+\frac{1}{2} v_{1} v_{2} \lambda_{5}\left(2 \cos ^{2} \theta-1\right)\right] \\
& =-v_{1}^{2} v_{2}^{2} \lambda_{5}\left[\cos ^{2} \theta+\frac{1}{2}\right] .
\end{aligned}
$$

Then

$$
\begin{aligned}
& V_{\theta}\left(v_{1}, v_{2}, \theta\right)<V_{\theta}\left(v_{1}, v_{2}, \stackrel{0}{\pi}\right) \Leftrightarrow \\
& -v_{1}^{2} v_{2}^{2} \lambda_{5}\left[\cos ^{2} \theta+\frac{1}{2}\right]<v_{1}^{2} v_{2}^{2} \lambda_{5}\left[\frac{1}{2} \mp 2 \cos \theta\right] \\
& \Leftrightarrow-\lambda_{5}(1 \mp \cos \theta)^{2}<0,
\end{aligned}
$$

that is $\lambda_{5}>0$.

\section{Rephasings}

The diagonalisation of the mass matrices $M_{d}^{0}$ and $M_{u}^{0}$ is only defined up to rephasings of the quark mass eigenstates. With

$$
\begin{aligned}
& M_{d}=\operatorname{diag}\left(m_{d_{j}}\right)=\mathcal{U}_{L}^{d \dagger} M_{d}^{0} \mathcal{U}_{R}^{d}, \\
& M_{u}=\operatorname{diag}\left(m_{u_{j}}\right)=\mathcal{U}_{L}^{u \dagger} M_{u}^{0} \mathcal{U}_{R}^{u},
\end{aligned}
$$

and the rephasings

$R_{d}=\operatorname{diag}\left(e^{i \varphi_{j}^{d}}\right), R_{u}=\operatorname{diag}\left(e^{i \varphi_{j}^{u}}\right), \quad R_{q}^{\dagger}=R_{q}^{-1}=R_{q}^{*}$,

it is clear that

$R_{d}^{\dagger} M_{d} R_{d}=M_{d}=\mathcal{U}_{L}^{d \dagger} M_{d}^{0} \mathcal{U}_{R}^{d}, \quad R_{u}^{\dagger} M_{u} R_{u}=M_{u}=\mathcal{U}_{L}^{u \dagger} M_{u}^{0} \mathcal{U}_{R}^{u}$.

Consequently the diagonalising unitary matrices $\mathcal{U}_{L}^{d}, \mathcal{U}_{L}^{u}, \mathcal{U}_{R}^{d}$ and $\mathcal{U}_{R}^{u}$ are only given up to common redefinitions

$\mathcal{U}_{L}^{d} \mapsto \mathcal{U}_{L}^{d} R_{d}, \mathcal{U}_{R}^{d} \mapsto \mathcal{U}_{R}^{d} R_{d}, \quad \mathcal{U}_{L}^{u} \mapsto \mathcal{U}_{L}^{u} R_{u}, \mathcal{U}_{R}^{u} \mapsto \mathcal{U}_{R}^{u} R_{u}$

Under such rephasings, the CKM matrix is transformed into

$V \mapsto R_{u}^{\dagger} V R_{d}, \quad V_{j k} \mapsto e^{i\left(\varphi_{k}^{d}-\varphi_{j}^{u}\right)} V_{j k}$
The off-diagonal elements of the matrices $N_{d}$ and $N_{u}$ are also transformed under rephasings,

$N_{d} \mapsto R_{d}^{\dagger} N_{d} R_{d}, \quad N_{u} \mapsto R_{u}^{\dagger} N_{u} R_{u}$,

with

$$
\begin{aligned}
& \hat{n}_{[\mathrm{d}] j}=\left[\mathcal{U}_{L}^{d}\right]_{3 j} \mapsto\left[\mathcal{U}_{L}^{d} R_{d}\right]_{3 j}=e^{i \varphi_{j}^{d}} \hat{n}_{[\mathrm{d}] j}, \\
& \hat{n}_{[\mathrm{d}] j}^{*} \hat{n}_{[\mathrm{d}] k} \mapsto e^{i\left(\varphi_{k}^{d}-\varphi_{j}^{d}\right)} \hat{n}_{[\mathrm{d}] j}^{*} \hat{n}_{[\mathrm{d}] k},
\end{aligned}
$$

and

$$
\begin{aligned}
& \hat{n}_{[\mathrm{u}] j}=\left[\mathcal{U}_{L}^{u}\right]_{3 j} \mapsto\left[\mathcal{U}_{L}^{u} R_{u}\right]_{3 j}=e^{i \varphi_{j}^{u}} \hat{n}_{[\mathrm{u}] j}, \\
& \hat{n}_{[\mathrm{u}] j}^{*} \hat{n}_{[\mathrm{u}] k} \mapsto e^{i\left(\varphi_{k}^{u}-\varphi_{j}^{u}\right)} \hat{n}_{[\mathrm{u}] j}^{*} \hat{n}_{[\mathrm{u}] k} .
\end{aligned}
$$

\section{One loop calculation of $\theta_{\mathrm{QFD}}$}

Following the paper of Goffin, Segre and Weldon (GSW) [36] we have

$$
\begin{aligned}
& \theta_{Q F D}=\frac{1}{16 \pi^{2}} \sum_{S=\mathrm{h}, \mathrm{H}, \mathrm{A}} \int_{0}^{1} d x \\
& \operatorname{Im} \operatorname{Tr}\left\{M^{-1} Y_{S} M^{\dagger} \ln \left[M M^{\dagger} x^{2}+m_{S}^{2}(1-x)\right] Y_{S}\right\}
\end{aligned}
$$

where

$M=\left(\begin{array}{cc}M_{d}^{0} & 0 \\ 0 & M_{u}^{0}\end{array}\right)$

With generalized Yukawa couplings defined by

$\mathscr{L}_{\mathrm{Y}}=\sum_{S=\mathrm{h}, \mathrm{H}, \mathrm{A}} S\left(\bar{d}_{L}^{0} Y_{S}^{0 d} d_{R}^{0}+\bar{u}_{L}^{0} Y_{S}^{0 u} u_{R}^{0}\right)$,

we have

$$
\begin{aligned}
& Y_{S}=\left(\begin{array}{cc}
Y_{S}^{0 d} & 0 \\
0 & Y_{S}^{0 u}
\end{array}\right), \\
& \text { where } \quad\left\{\begin{array}{l}
Y_{S}^{0 d}=-\frac{1}{v^{2}}\left[\mathcal{R}_{1 s} M_{d}^{0}+\left(\mathcal{R}_{2 s}+i \mathcal{R}_{3 s}\right) N_{d}^{0}\right], \\
Y_{S}^{0 u}=-\frac{1}{v^{2}}\left[\mathcal{R}_{1 s} M_{u}^{0}+\left(\mathcal{R}_{2 s}-i \mathcal{R}_{3 s}\right) N_{u}^{0}\right] .
\end{array}\right.
\end{aligned}
$$

$s=1,2,3$ corresponds to $\mathrm{h}, \mathrm{H}, \mathrm{A}$ respectively. Note that these imaginary pieces $i R_{3 s}$ will give the one loop contribution to $\theta_{\mathrm{QFD}}$. In Eq. (132) we can go to the weak basis where $M_{d}^{0}$ is diagonal, and therefore

$$
\begin{aligned}
M_{d}^{0}=V M_{d}, & N_{d}^{0}=V\left[t_{\beta} \mathbf{1}-\left(t_{\beta}+t_{\beta}^{-1}\right) \mathrm{P}_{d}\right] M_{d} \\
M_{u}^{0}=M_{u}, & N_{u}^{0}=\left[t_{\beta} \mathbf{1}-\left(t_{\beta}+t_{\beta}^{-1}\right) \mathrm{P}_{u}\right] M_{u} .
\end{aligned}
$$

$M_{d}$ and $M_{u}$ are the diagonal mass matrices, $V$ is the CKM matrix, and $\mathrm{P}_{d}=\mathcal{O}_{L}^{d T} \mathrm{P}_{3} \mathcal{O}_{L}^{d}$ and $\mathrm{P}_{u}=\mathcal{O}_{L}^{u T} \mathrm{P}_{3} \mathcal{O}_{L}^{u}$ are the projectors in Eqs.(23)-(27). The argument of the logarithm 
in Eq. (132) is $M M^{\dagger} x^{2}+m_{S}^{2}(1-x)$, which can be rewritten as

$\widetilde{V}\left[D D^{\dagger} x^{2}+m_{S}^{2}(1-x)\right] \widetilde{V}^{\dagger}$,

with block diagonal $\widetilde{V}$ and diagonal $D D^{\dagger}$ :

$\widetilde{V}=\left(\begin{array}{ll}V & 0 \\ 0 & 1\end{array}\right), \quad D D^{\dagger}=\left(\begin{array}{cc}M_{d}^{2} & 0 \\ 0 & M_{u}^{2}\end{array}\right)$,

and thus

$$
\ln \left[M M^{\dagger} x^{2}+m_{S}^{2}(1-x)\right]=\widetilde{V} \ln \left[D D^{\dagger} x^{2}+m_{S}^{2}(1-x)\right] \tilde{V}^{\dagger} .
$$

Note that all the matrices appearing in Eq. (132) are block diagonal, what can be traced back to the fact that charged Higgs do not contribute because there is not a second scale to generate dimensionless contributions as it should, see [36].

With

$F(a) \equiv \int_{0}^{1} d x \ln \left[a x^{2}+(1-x)\right]$,

we have

$$
\begin{aligned}
F(a)= & \ln a-2-\frac{1+\sqrt{1-4 a}}{2 a} \ln \left(\frac{1+\sqrt{1-4 a}-2 a}{1+\sqrt{1-4 a}}\right) \\
& -\frac{1-\sqrt{1-4 a}}{2 a} \ln \left(\frac{1-\sqrt{1-4 a}-2 a}{1-\sqrt{1-4 a}}\right), \quad(141)
\end{aligned}
$$

and thus

$$
\int_{0}^{1} d x\left(\ln \left[D D^{\dagger} x^{2}+m_{S}^{2}(1-x)\right]\right)_{q q^{\prime}}=L_{q}(S) \delta_{q q^{\prime}}
$$

where

$L_{q}(S)=\ln m_{S}^{2}+F\left(a_{S}^{q}\right) \quad$ and $\quad a_{S}^{q}=\left(\frac{m_{q}}{m_{S}}\right)^{2}$.

$F(0)=-1$ is a very good approximation, to be used in $L_{q}(S)$ for all quarks except $t . F(a)$ is plotted in Fig. 12; if all the scalars are heavier than the SM-like $\mathrm{h}$, then the maximal departure of $a_{S}^{q}$ from zero corresponds to $a_{\mathrm{h}}^{t}=\left(m_{t} / m_{\mathrm{h}}\right)^{2} \sim$ 1.9 , which gives $F(1.9) \sim 0.1$. With all the matrices block diagonal, we can split the up and down contributions

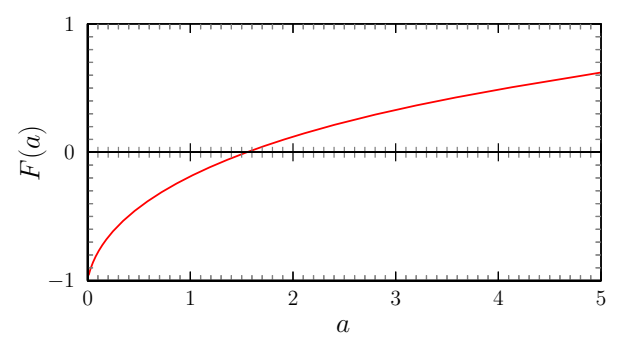

Fig. 12 Function $F(a)$ in Eq. (141)

$$
\begin{aligned}
\theta_{\mathrm{QFD}} & =\frac{1}{16 \pi^{2}} \sum_{s=\mathrm{h}, \mathrm{H}, \mathrm{A}} \operatorname{Im} \operatorname{Tr}\left\{M^{-1} Y_{S} M^{\dagger} \tilde{V} \mathrm{~L}(S) \widetilde{V}^{\dagger} Y_{S}\right\} \\
& =\theta_{\mathrm{QFD}}^{(d)}+\theta_{\mathrm{QFD}}^{(u)}
\end{aligned}
$$

where the diagonal matrix $\mathrm{L}(S)$

$\mathrm{L}(S)=\left(\begin{array}{cc}\mathrm{L}^{(d)}(S) & 0 \\ 0 & \mathrm{~L}^{(u)}(S)\end{array}\right)$

has elements $\left[\mathrm{L}^{(Q)}(S)\right]_{q q}=L_{q}(S)$.

Following Eqs. (135) and (136) we have

$Y_{S}^{d}=V\left[\alpha_{S}^{d} M_{d}+\beta_{S}^{d} \mathrm{P}_{d} M_{d}\right], \quad Y_{S}^{u}=\left[\alpha_{S}^{u} M_{u}+\beta_{S}^{u} \mathrm{P}_{u} M_{u}\right]$,

with

$\alpha_{S}^{d}=-\frac{1}{v}\left[\mathcal{R}_{1 s}+t_{\beta}\left(\mathcal{R}_{2 s}+i \mathcal{R}_{3 s}\right)\right]$,

$\alpha_{S}^{u}=-\frac{1}{v}\left[\mathcal{R}_{1 s}+t_{\beta}\left(\mathcal{R}_{2 s}-i \mathcal{R}_{3 s}\right)\right]$,

$\beta_{S}^{d}=\frac{1}{v}\left(t_{\beta}+t_{\beta}^{-1}\right)\left(\mathcal{R}_{2 s}+i \mathcal{R}_{3 s}\right)$,

$\beta_{S}^{u}=\frac{1}{v}\left(t_{\beta}+t_{\beta}^{-1}\right)\left(\mathcal{R}_{2 s}-i \mathcal{R}_{3 s}\right)$.

In general we have

$\theta_{\mathrm{QFD}}^{(u)}+\theta_{\mathrm{QFD}}^{(d)}=\frac{1}{16 \pi^{2}} \sum_{s=\mathrm{h}, \mathrm{H}, \mathrm{A}}\left(\operatorname{Im}\left[I_{S}^{(u)}\right]+\operatorname{Im}\left[I_{S}^{(d)}\right]\right)$,

where

$$
\begin{aligned}
I_{S}^{(q)} & =\operatorname{Tr}\left\{M_{q}^{-1} Y_{S}^{q} M_{q}^{\dagger} \widetilde{V} \mathrm{~L}^{(q)}(S) \widetilde{V}^{\dagger} Y_{S}^{q}\right\} \\
& =\operatorname{Tr}\left\{\left(\alpha_{S}^{q}+\beta_{S}^{q} P_{q}\right)^{2} M_{q} M_{q}^{\dagger} \mathrm{L}^{(q)}(S)\right\}
\end{aligned}
$$

It is clear that all the matrix traces are real and thus $\operatorname{Im}\left[I_{S}^{(q)}\right]$ comes from the imaginary parts in $\alpha_{S}^{q}$ and $\beta_{S}^{q}$, which depend on $\mathcal{R}_{3 s}$ and are clearly sensitive to CP violation in the Higgs sector, as expected. With

$\left(\alpha_{S}^{q}+\beta_{S}^{q} \mathrm{P}_{q}\right)^{2}=\left(\alpha_{S}^{q}\right)^{2}+\beta_{S}^{q}\left(2 \alpha_{S}^{q}+\beta_{S}^{q}\right) \mathrm{P}_{q}$,

we have

$\operatorname{Im}\left[\left(\alpha_{S}^{u}\right)^{2}\right]=-\frac{2}{v} t_{\beta}\left(\mathcal{R}_{1 s}+t_{\beta} \mathcal{R}_{2 s}\right) \mathcal{R}_{3 s}$,

$\operatorname{Im}\left[\beta_{S}^{u}\left(2 \alpha_{S}^{u}+\beta_{S}^{u}\right)\right]=\frac{2}{v}\left(t_{\beta}+t_{\beta}^{-1}\right)\left(\mathcal{R}_{1 s} \mathcal{R}_{3 s}+\left(t_{\beta}-t_{\beta}^{-1}\right) \mathcal{R}_{2 s} \mathcal{R}_{3 s}\right)$.

For $\alpha_{S}^{u}, \beta_{S}^{u} \rightarrow \alpha_{S}^{d}, \beta_{S}^{d}$, there is an overall minus sign arising from $\pm i \mathcal{R}_{3 s}$ in Eq. (147). Being the result proportional to 
$\operatorname{Tr}\left\{M_{q} M_{q}^{\dagger} \mathrm{L}^{(q)}(S)\right\}$ or to $\operatorname{Tr}\left\{\mathrm{P}_{q} M_{q} M_{q}^{\dagger} \mathrm{L}^{(q)}(S)\right\}$, the dominant piece will go as $m_{q_{i}}^{2}$ or $m_{q}^{2}\left(\hat{r}_{[\mathrm{q}] i}\right)^{2}$, and of course the leading contribution will come from the top quark

$$
\begin{aligned}
16 \pi^{2} \theta_{\mathrm{QFD}}^{t}= & -\frac{2 m_{t}^{2}}{v^{2}} t_{\beta}\left(\Delta_{13}^{t}+t_{\beta} \Delta_{23}^{t}\right)-\frac{2 m_{t}^{2}\left(\hat{r}_{[\mathrm{u}] 3}\right)^{2}}{v^{2}}\left(t_{\beta}+t_{\beta}^{-1}\right) \\
& {\left[\left({ }^{-1}-t_{\beta}\right) \Delta_{23}^{t}-\Delta_{13}^{t}\right] }
\end{aligned}
$$

with

$$
\begin{aligned}
& \Delta_{13}^{q}=\mathcal{R}_{12} \mathcal{R}_{32} J\left(\frac{m_{\mathrm{H}}^{2}}{m_{\mathrm{h}}^{2}}, \frac{m_{q}^{2}}{m_{\mathrm{h}}^{2}}\right)+\mathcal{R}_{13} \mathcal{R}_{33} J\left(\frac{m_{\mathrm{A}}^{2}}{m_{\mathrm{h}}^{2}}, \frac{m_{q}^{2}}{m_{\mathrm{h}}^{2}}\right), \\
& \Delta_{23}^{q}=\mathcal{R}_{22} \mathcal{R}_{32} J\left(\frac{m_{\mathrm{H}}^{2}}{m_{\mathrm{h}}^{2}}, \frac{m_{q}^{2}}{m_{\mathrm{h}}^{2}}\right)+\mathcal{R}_{23} \mathcal{R}_{33} J\left(\frac{m_{\mathrm{A}}^{2}}{m_{\mathrm{h}}^{2}}, \frac{m_{q}^{2}}{m_{\mathrm{h}}^{2}}\right),
\end{aligned}
$$

and

$$
J\left(\frac{m_{S}^{2}}{m_{\mathrm{h}}^{2}}, \frac{m_{q}^{2}}{m_{\mathrm{h}}^{2}}\right)=\ln \left(\frac{m_{S}^{2}}{m_{\mathrm{h}}^{2}}\right)+F\left(\frac{m_{q}^{2}}{m_{S}^{2}}\right)-F\left(\frac{m_{q}^{2}}{m_{\mathrm{h}}^{2}}\right),
$$

where orthogonality of the neutral Higgs mixing matrix has been used. It is after this GIM-type cancellation that the result does not depend on absolute scales. Note that if there is no $\mathrm{CP}$ violation in the Higgs sector $\mathcal{R}_{13}=\mathcal{R}_{23}=\mathcal{R}_{31}=$ $\mathcal{R}_{32}=0$ and then $\Delta_{13}^{q}=\Delta_{23}^{q}=0$ giving $\theta_{Q F D}^{t}=0$ as it should. Similar contributions - with a minus sign for down type quarks - can be written for lighter quarks, they are more naturally suppressed by $\left(m_{q} / v\right)^{2}$. The next contribution comes from the lighter $b$ and $c$ quarks that give

$$
\begin{aligned}
16 \pi^{2} \theta_{\mathrm{QFD}}^{\text {light }}= & \frac{2\left(m_{b}^{2}-m_{c}^{2}\right)}{v^{2}} t_{\beta}\left(\Delta_{13}+t_{\beta} \Delta_{23}\right) \\
+ & \frac{2\left(m_{b}^{2}\left(\hat{r}_{[\mathrm{d}] 3}\right)^{2}-m_{c}^{2}\left(\hat{r}_{[\mathrm{u}] 2}\right)^{2}\right)}{v^{2}}\left(t_{\beta}+t_{\beta}^{-1}\right) \\
& \times\left[\left(t_{\beta}^{-1}-t_{\beta}\right) \Delta_{23}-\Delta_{13}\right] .
\end{aligned}
$$

In $J$ we have approximated $\left(m_{q} / m_{S}\right)^{2}=0$, that is $J\left(m_{S}^{2} / m_{\mathrm{h}}^{2}, 0\right)=\ln \left(m_{S}^{2} / m_{\mathrm{h}}^{2}\right)$, and then the quark mass dependence in $\Delta_{13}^{q}$ and $\Delta_{23}^{q}$ disappears and we can write together the bottom and charm contributions. Other light quark contributions can be neglected.

From this detailed analysis of the different contributions, one could explore which regions of parameters are favoured if $\theta_{\mathrm{QFD}}$ below the $10^{-10}$ level is required. As anticipated in Sect. 6.1, the red regions in the different plots of Sect. 6.2 correspond to regions in parameter space which fulfill that requirement.

\section{References}

1. T. Lee, A theory of spontaneous T violation. Phys. Rev. D 8, 12261239 (1973)

2. G. Branco, P. Ferreira, L. Lavoura, M. Rebelo, M. Sher et al., Theory and phenomenology of two-Higgs-doublet models. Phys. Rep. 516, 1-102 (2012). arXiv:1106.0034

3. I.P. Ivanov, Building and testing models with extended Higgs sectors. Prog. Part. Nucl. Phys. 95, 160-208 (2017). arXiv: 1702.03776

4. S.L. Glashow, S. Weinberg, Natural conservation laws for neutral currents. Phys. Rev. D 15, 1958 (1977)

5. G. Branco, W. Grimus, L. Lavoura, Relating the scalar flavor changing neutral couplings to the CKM matrix. Phys. Lett. B 380, 119 126 (1996). arXiv:hep-ph/9601383

6. F. Botella, G. Branco, M. Rebelo, Minimal flavour violation and multi-Higgs models. Phys. Lett. B 687, 194-200 (2010). arXiv:0911.1753

7. F. Botella, G. Branco, M. Nebot, M. Rebelo, Two-Higgs leptonic minimal flavour violation. JHEP 1110, 037 (2011). arXiv: 1102.0520

8. G. Bhattacharyya, D. Das, A. Kundu, Feasibility of light scalars in a class of two-Higgs-doublet models and their decay signatures. Phys. Rev. D 89, 095029 (2014). arXiv:1402.0364

9. F. Botella, G. Branco, A. Carmona, M. Nebot, L. Pedro, M. Rebelo, Physical constraints on a class of Two-Higgs doublet models with FCNC at tree level. JHEP 1407, 078 (2014). arXiv:1401.6147

10. A. Celis, J. Fuentes-Martin, H. Serodio, An invisible axion model with controlled FCNCs at tree level. Phys. Lett. B 741, 117-123 (2015). arXiv:1410.6217

11. F.J. Botella, G.C. Branco, M. Nebot, M.N. Rebelo, Flavour changing Higgs couplings in a class of two Higgs doublet models. Eur. Phys. J. C 76(3), 161 (2016). arXiv:1508.05101

12. J.M. Alves, F.J. Botella, G.C. Branco, F. Cornet-Gomez, M. Nebot, Controlled flavour changing neutral couplings in two Higgs doublet models. Eur. Phys. J. C 77(9), 585 (2017). arXiv:1703.03796

13. G.C. Branco, Spontaneous CP nonconservation and natural flavor conservation: a minimal model. Phys. Rev. D 22, 2901 (1980)

14. G.C. Branco, M.N. Rebelo, The Higgs mass in a model with two scalar doublets and spontaneous CP violation. Phys. Lett. 160B, $117-120(1985)$

15. G.C. Branco, I.P. Ivanov, Group-theoretic restrictions on generation of CP-violation in multi-Higgs-doublet models. JHEP 01, 116 (2016). arXiv: 1511.02764

16. M. Dine, P. Draper, Challenges for the Nelson-Barr mechanism. JHEP 1508, 132 (2015). arXiv: 1506.05433

17. F. Botella, G. Branco, M. Nebot, M. Rebelo, New physics and evidence for a complex CKM. Nucl. Phys. B 725, 155-172 (2005). arXiv:hep-ph/0502133

18. H. Georgi, D.V. Nanopoulos, Suppression of flavor changing effects from neutral spinless meson exchange in gauge theories. Phys. Lett. B 82, 95 (1979)

19. J.F. Donoghue, L.F. Li, Properties of charged Higgs bosons. Phys. Rev. D 19, 945 (1979)

20. F.J. Botella, J.P. Silva, Jarlskog: like invariants for theories with scalars and fermions. Phys. Rev. D 51, 3870-3875 (1995). arXiv:hep-ph/9411288

21. P.M. Ferreira, L. Lavoura, J.P. Silva, A soft origin for CKM-type CP violation. Phys. Lett. B 704, 179-188 (2011). arXiv:1102.0784

22. G.C. Branco, Spontaneous CP violation in theories with more than four quarks. Phys. Rev. Lett. 44, 504 (1980)

23. Particle Data Group Collaboration, C. Patrignani et al., Review of Particle Physics. Chin. Phys. C40(10), 100001 (2016)

24. G. 't Hooft, Symmetry breaking through Bell-Jackiw anomalies. Phys. Rev. Lett. 37, 8-11 (1976) 
25. R.J. Crewther, P. Di Vecchia, G. Veneziano, E. Witten, Chiral estimate of the electric dipole moment of the neutron in quantum chromodynamics. Phys. Lett. 88B, 123 (1979). (Erratum: Phys. Lett.91B,487(1980))

26. M. Pospelov, A. Ritz, Electric dipole moments as probes of new physics. Ann. Phys. 318, 119-169 (2005). arXiv:hep-ph/0504231

27. R.D. Peccei, H.R. Quinn, CP conservation in the presence of instantons. Phys. Rev. Lett. 38, 1440-1443 (1977)

28. R.D. Peccei, H.R. Quinn, Constraints imposed by CP conservation in the presence of instantons. Phys. Rev. D 16, 1791-1797 (1977)

29. S. Weinberg, A new light boson? Phys. Rev. Lett. 40, 223-226 (1978)

30. F. Wilczek, Problem of strong $P$ and $T$ invariance in the presence of instantons. Phys. Rev. Lett. 40, 279-282 (1978)

31. M.A.B. Beg, H.S. Tsao, Strong P, T noninvariances in a superweak theory. Phys. Rev. Lett. 41, 278 (1978)

32. R.N. Mohapatra, G. Senjanovic, Natural suppression of strong P and T noninvariance. Phys. Lett. 79B, 283-286 (1978)

33. S.M. Barr, Solving the strong CP problem without the PecceiQuinn symmetry. Phys. Rev. Lett. 53, 329 (1984)

34. A.E. Nelson, Naturally weak CP violation. Phys. Lett. 136B, $387-$ 391 (1984)

35. L. Bento, G.C. Branco, P.A. Parada, A minimal model with natural suppression of strong CP violation. Phys. Lett. B 267, 95-99 (1991)

36. V. Goffin, G. Segre, H.A. Weldon, Explicit one loop corrections to the strong CP violating phase in $\mathrm{SU}(2)_{L} \times \mathrm{U}(1)$. Phys. Rev. D 21, $1410(1980)$

37. L.J. Hall, K. Harigaya, Implications of Higgs Discovery for the Strong CP Problem and Unification, arXiv: 1803.08119

38. W. Grimus, L. Lavoura, O. Ogreid, P. Osland, The oblique parameters in multi-Higgs-doublet models. Nucl. Phys. B 801, 81-96 (2008). arXiv:0802.4353

39. ATLAS, CMS Collaboration, G. Aad et al., Measurements of the Higgs boson production and decay rates and constraints on its couplings from a combined ATLAS and CMS analysis of the LHC pp collision data at $\sqrt{s}=7$ and 8 TeV. JHEP 08, 045 (2016). arXiv: 1606.02266

40. M. Nebot, J.P. Silva, Self-cancellation of a scalar in neutral meson mixing and implications for the LHC. Phys. Rev. D 92(8), 085010 (2015). arXiv:1507.07941

41. M. Jung, A. Pich, Electric dipole moments in two-Higgs-doublet models. JHEP 04, 076 (2014). arXiv:1308.6283

42. ATLAS Collaboration, M. Aaboud et al., Evidence for the $H \rightarrow$ $b \bar{b}$ decay with the ATLAS detector, JHEP 12, 024 (2017). arXiv: 1708.03299

43. CMS Collaboration, A.M. Sirunyan et al., Evidence for the Higgs boson decay to a bottom quark-antiquark pair, arXiv:1709.07497

44. F.J. Botella, F. Cornet-Gomez, M. Nebot, Flavor conservation in two-Higgs-doublet models. Phys. Rev. D 98(3), 035046 (2018). arXiv: 1803.08521

45. Heavy Flavor Averaging Group (HFAG) Collaboration, Y. Amhis et al., Averages of $b$-hadron, $c$-hadron, and $\tau$-lepton properties as of summer 2014, arXiv:1412.7515

46. M. Ciuchini, E. Franco, V. Lubicz, G. Martinelli, I. Scimemi, L. Silvestrini, Next-to-leading order QCD corrections to Delta $\mathrm{F}=2$ effective Hamiltonians. Nucl. Phys. B 523, 501-525 (1998). arXiv:hep-ph/9711402

47. A.J. Buras, M. Misiak, J. Urban, Two loop QCD anomalous dimensions of flavor changing four quark operators within and beyond the standard model. Nucl. Phys. B 586, 397-426 (2000). arXiv:hep-ph/0005183

48. J. Aebischer, M. Fael, C. Greub, J. Virto, B physics beyond the Standard Model at one loop: complete renormalization group evolution below the electroweak scale. JHEP 09, 158 (2017). arXiv: 1704.06639
49. ETM Collaboration, N. Carrasco, P. Dimopoulos, R. Frezzotti, V. Lubicz, G.C. Rossi, S. Simula, C. Tarantino, $\Delta S=2$ and $\Delta C=2$ bag parameters in the standard model and beyond from $N_{f}=$ $2+1+1$ twisted-mass lattice QCD. Phys. Rev. D 92(3), 034516 (2015). arXiv: 1505.06639

50. Fermilab Lattice, MILC Collaboration, A. Bazavov et al., $B_{(s)}^{0}$ mixing matrix elements from lattice QCD for the Standard Model and beyond. Phys. Rev. D 93(11), 113016 (2016). arXiv: 1602.03560

51. S. Aoki et al., Review of lattice results concerning low-energy particle physics. Eur. Phys. J. C 77(2), 112 (2017). arXiv:1607.00299

52. M. Misiak et al., Estimate of $\mathcal{B}\left(\bar{B} \rightarrow X_{s} \gamma\right)$ at $O\left(\alpha_{s}^{2}\right)$. Phys. Rev. Lett. 98, 022002 (2007). arXiv:hep-ph/0609232

53. A. Crivellin, A. Kokulu, C. Greub, Flavor-phenomenology of twoHiggs-doublet models with generic Yukawa structure. Phys. Rev. D 87(9), 094031 (2013). arXiv:1303.5877

54. ATLAS Collaboration, M. Aaboud et al., Search for top quark decays $t \rightarrow q H$, with $H \rightarrow \gamma \gamma$, in $\sqrt{s}=13 \mathrm{TeV} p p$ collisions using the ATLAS detector. JHEP 10, 129 (2017). arXiv:1707.01404

55. CMS Collaboration, V. Khachatryan et al., Search for top quark decays via Higgs-boson-mediated flavor-changing neutral currents in pp collisions at $\sqrt{s}=8 \mathrm{TeV}$. JHEP 02, 079 (2017). arXiv: 1610.04857

56. CMS Collaboration, A.M. Sirunyan et al., Search for the flavorchanging neutral current interactions of the top quark and the Higgs boson which decays into a pair of b quarks at $\sqrt{s}=13 \mathrm{TeV}$, arXiv: 1712.02399

57. D. Barducci, A.J. Helmboldt, Quark flavour-violating Higgs decays at the ILC. JHEP 12, 105 (2017). arXiv:1710.06657

58. HFLAV Collaboration, Y. Amhis et al., Averages of $b$-hadron, $c$ hadron, and $\tau$-lepton properties as of summer 2016. Eur. Phys. J. C 77(12), 895 (2017). arXiv:1612.07233

59. J. Albrecht, S. Reichert, D. van Dyk, Status of rare exclusive $B$ meson decays in 2018, arXiv: 1806.05010

60. P. Bechtle, S. Heinemeyer, O. Stal, T. Stefaniak, G. Weiglein, Applying exclusion likelihoods from LHC searches to extended Higgs sectors. Eur. Phys. J. C 75(9), 421 (2015). arXiv:1507.06706

61. I.P. Ivanov, J.P. Silva, Tree-level metastability bounds for the most general two Higgs doublet model. Phys. Rev. D 92(5), 055017 (2015). arXiv: 1507.05100

62. I.P. Ivanov, Minkowski space structure of the Higgs potential in 2HDM. II. Minima, symmetries, and topology. Phys. Rev. D 77, 015017 (2008). arXiv:0710.3490

63. A. Barroso, P.M. Ferreira, I.P. Ivanov, R. Santos, J.P. Silva, Evading death by vacuum. Eur. Phys. J. C 73, 2537 (2013). arXiv:1211.6119

64. A. Barroso, P.M. Ferreira, I.P. Ivanov, R. Santos, Metastability bounds on the two Higgs doublet model. JHEP 06, 045 (2013). arXiv: 1303.5098

65. S. Kanemura, T. Kubota, E. Takasugi, Lee-Quigg-Thacker bounds for Higgs boson masses in a two doublet model. Phys. Lett. B 313, 155-160 (1993). arXiv:hep-ph/9303263

66. I.F. Ginzburg, I.P. Ivanov, Tree-level unitarity constraints in the most general 2HDM. Phys. Rev. D 72, 115010 (2005). arXiv:hep-ph/0508020

67. B. Grinstein, C.W. Murphy, P. Uttayarat, One-loop corrections to the perturbative unitarity bounds in the CP-conserving two-Higgs doublet model with a softly broken $\mathbb{Z}_{2}$ symmetry. JHEP 06, 070 (2016). arXiv: 1512.04567 